\title{
Waves, bumps, and patterns in neural field theories
}

\author{
S Coombes*
}

April 13, 2005

\begin{abstract}
Neural field models of firing rate activity have had a major impact in helping to develop an understanding of the dynamics seen in brain slice preparations. These models typically take the form of integrodifferential equations. Their non-local nature has led to the development of a set of analytical and numerical tools for the study of waves, bumps and patterns, based around natural extensions of those used for local differential equation models. In this paper we present a review of such techniques and show how recent advances have opened the way for future studies of neural fields in both one and two dimensions that can incorporate realistic forms of axo-dendritic interactions and the slow intrinsic currents that underlie bursting behaviour in single neurons.
\end{abstract}

\section{Introduction}

The multi-scale properties of spatio-temporal neural activity leads naturally to some interesting mathematical challenges, in terms of both modelling strategies and subsequent analysis. Since the number of neurons and synapses in even a small piece of cortex is immense a popular modelling approach has been to take a continuum limit and study neural networks in which space is continuous and macroscopic state variables are mean firing rates. Perhaps the first attempt at developing a continuum approximation of neural activity can be attributed to Beurle [1] in the 1950's and later by Griffith $[2,3]$ in the 1960's. By focusing

\footnotetext{
${ }^{*}$ Department of Mathematical Sciences, University of Nottingham, Nottingham, NG7 2RD, UK. email: stephen. coombes@nottingham.ac.uk
}

on the proportion of neurons becoming activated per unit time in a given volume of model brain tissue consisting of randomly connected neurons, Beurle was able to analyse the triggering and propagation of large scale brain activity. However, this work only dealt with networks of excitatory neurons with no refractory or recovery variable. It was Wilson and Cowan in the 1970's [4, 5] who extended Beurles work to include both inhibitory and excitatory neurons as well as refractoriness. For a fascinating historical perspective on this work we refer the reader to the recent article by Cowan [6]. Further work, particularly on pattern formation, in continuum models of neural activity was pursued by Amari [7, 8] under natural assumptions on the connectivity and firing rate function. Amari considered local excitation and distal inhibition which is an effective model for a mixed population of interacting inhibitory and excitatory neurons with typical cortical connections (commonly referred to as Mexican hat connectivity). Since these seminal contributions to dynamic neural field theory similar models have been used to investigate EEG rhythms [9], visual hallucinations [10, 11], mechanisms for short term memory $[12,13]$ and motion perception [14]. The sorts of dynamic behaviour that are typically observed in neural field models includes, spatially and temporally periodic patterns (beyond a Turing instability) $[10,15]$, localised regions of activity (bumps and multi-bumps) $[12,16]$ and travelling waves (fronts, pulses, target waves and spirals) $[17,18,19]$. In the latter case corresponding phenomena may be observed experimentally using multi-electrode recordings and imaging methods. In particular it is possible to electrically stimulate slices of pharmacologically treated tissue taken from the cortex [20,21, 22], hippocampus 
[23] and thalamus [24]. In brain slices these waves can take the form of spindle waves seen at the onset of sleep [25], the propagation of synchronous discharge during an epileptic seizure [26] and waves of excitation associated with sensory processing [27]. Interestingly, spatially localised bumps of activity have been linked to working memory (the temporary storage of information within the brain) in prefrontal cortex $[28,29]$, representations in the head-direction system [30], and feature selectivity in the visual cortex, where bump formation is related to the tuning of a particular neuron's response [31].

In this paper we present a review of neural field theories of Wilson-Cowan and Amari type and describe the mathematical techniques that have been used in their analysis to date. For the purposes of exposition we shall stick to single population models, though all of what we say can be easily taken over to the case of two or more populations, such as discussed in [32]. In section 2 we introduce the standard integrodifferential equation (IDE) for a scalar neural field and discuss the conditions under which this reduces to a local partial differential equation (PDE) model. Moreover, we show how the IDE model may be written as a purely integral equation. The integral framework is convenient for certain types of analysis, such as calculating the onset of a Turing instability. We briefly illustrate this in section 3 . Next in section 4 we move on to a study of travelling wave solutions and show how techniques from the reaction-diffusion literature may be used to provide estimates of wave speed. Interestingly for the choice of a Heaviside firing rate function wave speeds can be calculated exactly. We describe this procedure for travelling front solutions in section 5. Moreover, borrowing from ideas first developed in the PDE community we also show how to analyse wave stability using an Evans function approach. In section 6 we consider a slightly more general set of neural field equations that incorporate modulatory terms. These models support travelling pulses, as well as fronts. Generalising the techniques used for the study of fronts we show how to determine pulse speed and stability as a function of system parame- ters. Moreover, localised bump solutions are similarly handled by recognising them as standing pulse waves. The extension of the standard model to incorporate space-dependent delays, arising from axonal and dendritic communication delays is the subject of section 7. As well as describing the conditions under which these models may be reduced to a PDE description, we review the effect of such delays on the onset of a dynamic Turing instability. In section 8 we return to the starting point for the derivation of a firing rate model and show how it is also possible to carry forward slow ionic currents into a firing rate description of neural tissue. In illustration we construct a firing rate model that incorporates a slow T-type calcium current, $I_{T}$, known to be important in the bursting response of thalamo-cortical relay cells. Moreover, for a purely inhibitory network and a Heaviside firing rate function we show how to construct so-called lurching pulses, often seen in simulations of more detailed biophysical networks expressing $I_{T}$. Some non-trivial consequences of working with neural fields in two dimensions are discussed in section 9. In particular we show that the stable so-called dimple bump that can be found in a one dimensional model does not have a stable two dimensional analogue. Finally in section 10 we discuss some of the open challenges relating to the further development and analysis of neural field theories.

\section{Mathematical framework}

In many continuum models for the propagation of electrical activity in neural tissue it is assumed that the synaptic input current is a function of the pre-synaptic firing rate function [5]. These infinite dimensional dynamical systems are typically variations on the form [32]

$$
\frac{1}{\alpha} \frac{\partial u(x, t)}{\partial t}=-u+\int_{-\infty}^{\infty} \mathrm{d} y w(y) f \circ u(x-y, t) .
$$

Here, $u(x, t)$ is interpreted as a neural field representing the local activity of a population of neurons at position $x \in \mathbb{R}$. The second term on the right represents the synaptic input, with $f \circ u$ interpreted as the 
firing rate function. The strength of connections between neurons separated by a distance $y$ is denoted $w(y)=w(|y|)$ (assuming a spatially homogeneous and isotropic system), and is often referred to as the synaptic footprint.

There are several natural choices for the firing rate function, the simplest being a Heaviside step function. In this case a neuron fires maximally (at a rate set by its absolute refractory period) or not at all, depending on whether or not synaptic activity is above or below some threshold. In a statistical mechanics approach to formulating mean-field neural equations this all or nothing response is replaced by a smooth sigmoidal form [4, 33]. For an arbitrary firing rate response the model (1) is naturally written in the form

$$
Q u(x, t)=\psi(x, t)
$$

where $\psi(x, t)$ is given by the second term on the right hand side of (1) and $Q=\left(1+\alpha^{-1} \partial_{t}\right)$. The linear differential operator $Q$ is used to model the dynamics associated with first order synaptic processing and can easily be generalised to represent higher order synaptic processing [34]. It is convenient to write $\psi(x, t)$ in the form

$$
\psi(x, t)=(w \otimes f)(x, t),
$$

where $\otimes$ represents a spatial convolution:

$$
(w \otimes f)(x, t)=\int_{-\infty}^{\infty} w(y) f(x-y, t) \mathrm{d} y .
$$

Numerical simulations of (2) using the integral equation (3) with sigmoidal $f$, show that such systems support unattenuated travelling waves as a result of localised input [5]. We note that the time independent solutions of (2) are given by $u(x)=\psi(x)$. For this class of solutions Amari [8] was able to find localised stable pulses that are bistable with a homogeneous steady state, assuming a Heaviside firing rate function and Mexican hat connectivity. Subsequently Kishimoto and Amari showed that such solutions also exist for a smooth sigmoidal function (at least in the high gain limit) [16].

For certain (special) choices of $w(x)$ it is possible to re-cast the equation for $\psi(x, t)$ in a more local form [17]. If the Fourier transform, $\operatorname{FT}[w](k)=$
$\int_{\mathbb{R}} \mathrm{e}^{i k x} w(x) \mathrm{d} x$, of $w(x)$ has a simple rational polynomial structure we may exploit the convolution property of (3) to write the equation for $\psi(x, t)$ as a PDE. To illustrate this consider the choice $w(x)=\mathrm{e}^{-|x|} / 2$, with $\operatorname{FT}[w](k)=\left(1+k^{2}\right)^{-1}$. In this case taking the Fourier transform of (3) yields

$$
\operatorname{FT}[\psi](k, t)=\frac{1}{1+k^{2}} \operatorname{FT}[f \circ u](k, t) .
$$

Cross multiplying by $1+k^{2}$ and inverting (remembering that $\left.\operatorname{FT}\left[\psi_{x}\right](k)=i k \psi\right)$, gives

$$
\left(1-\partial_{x x}\right) \psi(x, t)=f \circ u(x, t) .
$$

Hence, the evolution of $u$ is described by the pair of coupled partial differential equations, (2) and (6). By exploiting the local PDE structure that can be obtained with such special choices it is possible to use many of the standard tools from dynamical systems analysis to study solutions of inherently non-local neural field models. For example, in a co-moving frame travelling wave solutions are given by a system of ordinary differential equations (ODEs), with travelling fronts and pulses viewed as global (heteroclinic and homoclinic) connections. Standard shooting and numerical continuation techniques (both numerical and analytical) may then be brought to bear in their construction $[18,34]$.

Another common choice of $w(x)$ in the study of neural field models is that of a Mexican hat function, such as $w(x)=(1-|x|) \mathrm{e}^{-|x|} / 4$ (perhaps more properly called a wizard hat function, because of its cusp at the origin [35]). In this case $\mathrm{FT}[w](k)=k^{2} /\left(1+k^{2}\right)^{2}$, and a similar argument to that above gives

$$
\left(1-\partial_{x x}\right)^{2} \psi(x, t)=-[f \circ u(x, t)]_{x x} .
$$

Time-independent solutions of (7) are solutions of the fourth order ODE: $\left(1-\mathrm{d}_{x x}\right)^{2} u(x, t)=-[f \circ u(x)]_{x x}$ (using $Q u(x)=\psi(x))$. Interestingly, numerical solution of such systems typically yield single and multi-bump structures (regions of localised activity) [12, 13]. Moreover, the governing equations are now known to possess a Hamiltonian structure [12]. The extensive use of local PDE methods (particularly those for fourth order reversible systems) for studying such localised 
structures can be found in the work of Laing and Troy [36] and Krisner [37]. A detailed numerical analysis of localised time-independent solutions to equation (7) with a sigmoidal form of firing rate function can be found in [34]. Here, it is shown that this particular fourth order system admits multiple bump solutions, and that such localised multi-bumps are lost (in favour of global patterns) when a stable $N$-bump and an unstable $(N+2)$-bump coalesce.

Apart from waves and bumps, neural field models are also known to support the formation of globally periodic patterns [10]. Such patterns can emerge beyond a so-called Turing bifurcation point. To develop this, and other techniques, in full generality, it is convenient to use the language of Green's functions and write

$$
Q \eta(t)=\delta(t),
$$

where $\eta(t)$ is the Green's function of the linear differential operator $Q$, with $\eta(t)=0$ for $t \leq 0$. For the first-order case considered till now $\left(Q=1+\alpha^{-1} \partial_{t}\right)$, the Green's function is simply $\eta(t)=\alpha \mathrm{e}^{-\alpha t}$. For the second order operator $Q=\left(1+\alpha^{-1} \partial_{t}\right)^{2}$, the Green's function is that of that of the often used alpha function, $\eta(t)=\alpha^{2} t \mathrm{e}^{-\alpha t}$. We are now in a position to deal with neural field models in a purely integral framework by integrating (2) to obtain

$$
u=\eta * w \otimes f \circ u
$$

where the temporal convolution $*$ is defined by

$$
(\eta * f)(x, t)=\int_{0}^{t} \eta(s) f(x, t-s) \mathrm{d} s .
$$

The distributed delay kernel $\eta(t)$ can be chosen so as best to describe the response of a given synapse.

\section{Turing instability analysis}

We now describe how a spatially homogeneous state can become unstable to spatially heterogeneous perturbations, resulting in the formation of periodic patterns. We do this using a Turing instability analysis. One solution of the neural field equation (1) is the spatially uniform resting state $u(x, t)=\bar{u}$ for all $x, t$, de- fined by

$$
\bar{u}=\kappa f(\bar{u}),
$$

where $\kappa=\int_{\mathbb{R}} w(y) \mathrm{d} y$. Note that for positive weight kernels it is quite common to normalise them such that $\int_{\mathbb{R}} w(y) \mathrm{d} y=1$. We linearise about the steady state by letting $u(x, t) \rightarrow \bar{u}+u(x, t)$ so that $f(u) \rightarrow f(\bar{u})+$ $f^{\prime}(\bar{u}) u$ to obtain

$$
u=\gamma \eta * w \otimes u, \quad \gamma=f^{\prime}(\bar{u}) .
$$

This has solutions of the form $\mathrm{e}^{\lambda t} \mathrm{e}^{i k x}$, so that $\lambda=\lambda(k)$ is given implicitly by the solution to

$$
\gamma \operatorname{LT}[\eta](\lambda) \operatorname{FT}[w](k)-1=0,
$$

where $\operatorname{LT}[\eta](\lambda)$ is the Laplace transform of $\eta(t)$ :

$$
\operatorname{LT}[\eta](\lambda)=\int_{0}^{\infty} \eta(s) \mathrm{e}^{-\lambda s} \mathrm{~d} s .
$$

The uniform steady state is linearly stable if $\operatorname{Re} \lambda(k)<$ 0 for all $k \in \mathbb{R}, k \neq 0$. For example, with an exponential synapse $\eta(t)=\mathrm{e}^{-t}$, the stability condition is simply

$$
\operatorname{FT}[w](k)<\gamma^{-1} \quad \text { for all } k \in \mathbb{R}, \quad k \neq 0 .
$$

Now consider the case that $\mathrm{FT}[w](k)$ has a positive maximum $\widehat{w}_{\max }$ at $k= \pm k_{c}$, that is $\operatorname{FT}[w]\left(k_{c}\right)=\widehat{w}_{\max }$ and $\operatorname{FT}[w](k)<\widehat{w}_{\max }$ for all $k \neq k_{c}$. Let $\gamma_{c}^{-1}=\widehat{w}_{\max }$, then (i) For $\gamma<\gamma_{c}$ the resting state is linearly stable. (ii) At the critical point $\gamma=\gamma_{c}$ there is an instability due to excitation of the pattern $\mathrm{e}^{ \pm i k_{c} x}$. (iii) Beyond the bifurcation point, $\gamma>\gamma_{c}, \lambda\left(k_{c}\right)>0$ and this pattern grows with time. In fact there will typically exist a range of values of $k \in\left(k_{1}, k_{2}\right)$ for which $\lambda(k)>0$, signalling a set of growing patterns. As the patterns grow, the linear approximation breaks down and nonlinear terms dominate behaviour. (iv) The saturating property of $f(u)$ tends to create patterns with finite amplitude, that scale as $\sqrt{k-k_{c}}$ close to bifurcation and have wavelength $2 \pi / k_{c}$. (v) If $k_{c}=0$ then we would have a bulk instability resulting in the formation of another homogeneous state. Note that if $\operatorname{Im} \lambda\left(k_{c}\right) \neq 0$, then the homogeneous solution would be time-periodic.

Since the Fourier transform of Mexican hat type functions, which represent short-range excitation and 
long range inhibition, are peaked away from the origin they are capable of supporting a Turing instability. An example of such a function is $w(x)=e^{-|x|}$ $e^{-|x| / 2} / 2$ (a wizard hat). Another classic example is a difference of Gaussians.

Generalising this approach to two dimensions is straight forward. Near a bifurcation point we would expect spatially heterogeneous solutions of the form $\mathrm{e}^{\lambda t} \mathrm{e}^{i \mathbf{k}_{c} \cdot \mathbf{r}}$ for some $\mathbf{k}_{c}=\left(k_{1}, k_{2}\right)$, and $\mathbf{r} \in \mathbb{R}^{2}$. For a given $k_{c}=\left|\mathbf{k}_{c}\right|$ there are an infinite number of choices for $k_{1}$ and $k_{2}$. It is therefore common to restrict attention to doubly periodic solutions that tessellate the plane. These can be expressed in terms of the basic symmetry groups of hexagon, square and rhombus. Solutions can then be constructed from combinations of the basic functions $\mathrm{e}^{i k_{c} \mathbf{R} \cdot \mathbf{r}}$, for appropriate choices of the basis vectors R. Details of this programme, and the nonlinear analysis necessary in order to correctly select which of the modes will stably appear are discussed in $[10,15,32,38,39]$. For a recent discussion of how to treat spatio-temporal pattern formation in systems with heterogeneous connection topologies (more realistic of real cortical structures) we refer the reader to $[40,41]$.

\section{Travelling waves}

Waves in the form of travelling fronts and pulses have now been observed in a variety of slice preparations [20, 21, 22, 42, 43]. To establish properties of waves in a model neural system it is convenient to introduce the coordinate $\xi=x-c t$ and seek functions $U(\xi, t)=$ $u(x-c t, t)$ that satisfy $(9)$. In the $(\xi, t)$ coordinates, the integral equation (9) reads

$$
\begin{aligned}
U(\xi, t) & =\int_{-\infty}^{\infty} \mathrm{d} y w(y) \int_{0}^{\infty} \mathrm{d} s \eta(s) \\
& \times f \circ U(\xi-y+c s, t-s) .
\end{aligned}
$$

A travelling wave, with speed $c$, is a stationary solution $U(\xi, t)=q(\xi)$ (independent of $t$ ), that satisfies

$$
q(\xi)=\int_{0}^{\infty} \eta(z) \psi(\xi+c z) \mathrm{d} z,
$$

with

$$
\psi(\xi)=\int_{-\infty}^{\infty} w(y) f(q(\xi-y)) \mathrm{d} y .
$$

Note that standing waves (with $c=0$ ), are defined by $Q u(x, t)=q(x)$ so that $q(x)=\psi(x)$. It is convenient to regard the bumps of spatially localised timeindependent solutions that we have mentioned earlier as standing waves with speed $c=0$.

For sigmoidal firing rate functions it is generally possible to arrange for the system to have three homogeneous steady states, $u_{1}<u_{2}<u_{3}$. In this case it is natural to look for travelling front solutions with $q(-\infty)=u_{3}$ and $q(\infty)=u_{1}$, which connect $u_{1}$ and $u_{3}$ (which are stable to homogeneous perturbations). Arguing in analogy to techniques used for estimating front speeds for reaction-diffusion equations we consider the linearised equations of motion around the fixed points. In this case we are led to consider systems with linear firing rate functions of the form $f(u)=\gamma u$, which give rise to exponential solutions $u(\xi) \sim \mathrm{e}^{\lambda \xi}$. It is easily established that $\lambda$ is the solution to $L(c, \lambda)=0$ where

$$
L(c, \lambda)=\gamma \operatorname{LT}[\eta](-c \lambda) \mathrm{FT}[w](-i \lambda)-1
$$

If the temporal and spatial kernels, $\eta(t)$ and $w(x)$, are both normalised to unity, then we see that that $L(c, 0)=\gamma-1$. For $\lambda<0, L(c, \lambda)$ is a monotonically decreasing function of $c$ with $\lim _{c \rightarrow \infty} L(c, \lambda)=-1$. Moreover, we have that

$$
\left.\frac{\partial L(c, \lambda)}{\partial \lambda}\right|_{\lambda=0}=\gamma c \int_{0}^{\infty} s \eta(s) \mathrm{d} s>0
$$

for $c>0$, and that $\partial^{2} L / \partial^{2} \lambda(c, \lambda)>0$ for all $\lambda$ and $c>0$ (i.e. $L(c, \lambda)$ is a convex function of $\lambda$ ). Following Diekmann $[44,45]$, we introduce a minimum propagation speed, $c^{*}$, as

$$
c^{*}=\inf \{c \mid L(c, \lambda)=0, \text { for some } \lambda<0\} .
$$

Now, choosing $\gamma>1$, a minimum with $c>0$ can only occur for $\lambda<0$ (using the convexity of $L(c, \lambda)$ and the fact that $\partial L / \partial \lambda(c, 0)>0)$. Consider, for example the case of an exponential synaptic footprint $w(x)=$ $\mathrm{e}^{-|x|} / 2$ and an exponential synapse $\eta(t)=\alpha \mathrm{e}^{-\alpha t}$. In this case we have from (19) that $c=c(\lambda)$, where

$$
c(\lambda)=\frac{\alpha}{\lambda}\left[1-\frac{\gamma}{1-\lambda^{2}}\right]
$$


for $|\lambda|<1$. Since the value of $\lambda$ for which $c^{\prime}(\lambda)=0$ is independent of $\alpha$ we immediately see that $c^{*}$ is linear in $\alpha$. For a general nonlinear firing rate $c^{*}$ is still expected to be a good predictor of wave speed if the linearisation at $u=\bar{u}$ satisfies $f(u)<f^{\prime}(\bar{u}) u[44,45]$.

For sigmoidal firing rate functions it has been shown by Ermentrout and McLeod that there exists a unique monotone travelling wave front for positive spatially decaying synaptic connections [17]. Indeed, there are now a number of results about existence, uniqueness and asymptotic stability of waves for IDEs, such as can be found in [8, 46, 47, 48]. Other work on the properties of travelling fronts, and in particular speed as a function of system parameters can be found in $[18,34,49]$. Note also that a formal link between travelling front solutions in neural field theories and travelling spikes in integrate-and-fire networks can be found in [50].

The linear stability of waves is obtained by writing $U(\xi, t)=q(\xi)+u(\xi) \mathrm{e}^{\lambda t}$, and Taylor expanding (16), to obtain the eigenvalue equation $u=\mathcal{L} u$ :

$$
\begin{aligned}
u(\xi) & =\int_{-\infty}^{\infty} \mathrm{d} y w(y) \int_{0}^{\infty} \mathrm{d} s \eta(s) \mathrm{e}^{-\lambda s} f^{\prime}(q(\xi-y+c s)) \\
& \times u(\xi-y+c s) .
\end{aligned}
$$

A travelling wave is said to be linearly stable if $\operatorname{Re}(\lambda)<0$ for $\lambda \neq 0$. Since we are concerned with systems where the real part of the continuous spectrum has a uniformly negative upper bound, it is enough to determine the location of the normal spectrum for wave stability [51]. In general the normal spectrum of the operator obtained by linearising a system about its travelling wave solution may be associated with the zeros of a complex analytic function, the so-called Evans function. This was originally formulated by Evans [52] in the context of a stability theorem about excitable nerve axon equations of Hodgkin-Huxley type. The extension to integral models is far more recent $[51,53,54,55,56,57]$.

Throughout the rest of this paper we shall focus on the particular choice of a Heaviside firing rate function, $f(u)=\Theta(u-h)$ for some threshold $h$. The Heaviside function is defined by $\Theta(x)=1$ for $x \geq 0$ and is zero otherwise. In this case $\psi$ depends only on where the threshold is crossed and not directly on the shape of $u$. Apart from allowing an explicit construction of travelling waves this choice also allows for a direct calculation of wave stability via the construction of an Evans function [51]. Although often chosen for mathematical reasons the Heaviside function may be regarded as a natural restriction of sigmoidal functions to the regime of high gain. Importantly, numerical simulations show that many of the qualitative properties of solutions in the high gain limit are retained with decreasing gain $[17,18,34]$.

\section{Fronts in a scalar integral model}

In this section we introduce the techniques for constructing the Evans function with the example of travelling front solutions to (9). A more detailed discussion of the construction of the Evans function for neural field theories can be found in [51]. We look for travelling front solutions such that $q(\xi)>h$ for $\xi<0$ and $q(\xi)<h$ for $\xi>0$. It is then a simple matter to show that

$$
\psi(\xi)=\int_{\xi}^{\infty} w(y) \mathrm{d} y
$$

The choice of origin, $q(0)=h$, gives an implicit equation for the speed of the wave as a function of system parameters.

The construction of the Evans function begins with an evaluation of (23). Using the identity

$$
\frac{\mathrm{d}}{\mathrm{d} q} \Theta(q(\xi)-h)=\frac{\delta(\xi)}{\left|q^{\prime}(0)\right|}
$$

we arrive at the expression

$$
u(\xi)=\frac{u(0)}{c\left|q^{\prime}(0)\right|} \int_{-\infty}^{\infty} \mathrm{d} y w(y) \eta(-\xi / c+y / c) \mathrm{e}^{-\lambda(y-\xi) / c}
$$

From this equation we may generate a self-consistent equation for the value of the perturbation at $\xi=0$, simply by setting $\xi=0$ on the left hand side of (26). This self-consistent condition reads

$$
u(0)=\frac{u(0)}{c\left|q^{\prime}(0)\right|} \int_{0}^{\infty} \mathrm{d} y w(y) \eta(y / c) \mathrm{e}^{-\lambda y / c},
$$

remembering that $\eta(t)=0$ for $t \leq 0$. Importantly this has a non-trivial solution if $\mathcal{E}(\lambda)=0$, where

$$
\mathcal{E}(\lambda)=1-\frac{1}{c\left|q^{\prime}(0)\right|} \int_{0}^{\infty} \mathrm{d} y w(y) \eta(y / c) \mathrm{e}^{-\lambda y / c} .
$$


We identify (28) with the Evans function for the travelling front solution of (9). It can be shown that i) the Evans function is only real-valued if the eigenvalue parameter $\lambda$ is real, ii) the complex number $\lambda$ is an eigenvalue of the operator $\mathcal{L}$ if and only if $\mathcal{E}(\lambda)=0$, and iii) the algebraic multiplicity of an eigenvalue is exactly equal to the order of the zero of the Evans function [51]. Also, from translation invariance, $\lambda=0$ is an eigenvalue (with eigenfunction $q^{\prime}(\xi)$ ), so that $\mathcal{E}(0)=0$.

A common choice for the synaptic response function is $\eta(t)=\alpha \mathrm{e}^{-\alpha t}$. In this case the condition $q(0)=h$ gives an implicit expression for the speed of the wave in the form [58]

$$
h=\frac{\kappa}{2}-\operatorname{LT}[w](\alpha / c) .
$$

Moreover, the Evans function takes the explicit form

$$
\mathcal{E}(\lambda)=1-\frac{\operatorname{LT}[w]((\alpha+\lambda) / c))}{\operatorname{LT}[w](\alpha / c)},
$$

where we have made use of the fact that $\mathcal{E}(0)=0$.

As an example it is illustrative to consider $w(x)=$ $\mathrm{e}^{-|x|} / 2$, with Laplace transform $\operatorname{LT}[w](\alpha)=(1+$ $\alpha)^{-1} / 2$. The speed of the front is determined from (29) as

$$
c=\alpha \frac{1-2 h}{2 h},
$$

which we observe is linear in $\alpha$ (as in the earlier example for a linear firing rate function). Using (30) the Evans function is easily calculated as

$$
\mathcal{E}(\lambda)=\frac{\lambda}{c+\alpha+\lambda} .
$$

The equation $\mathcal{E}(\lambda)=0$ only has the solution $\lambda=0$. We also have that $\mathcal{E}^{\prime}(0)>0$, showing that $\lambda=0$ is a simple eigenvalue. Hence, the travelling wave front for this example is linearly stable. Assuming $c>0$ the travelling front (17) is given in terms of (24) which takes the explicit form

$$
\psi(\xi)=\left\{\begin{array}{ll}
\frac{1}{2} \mathrm{e}^{-\xi} & \xi \geq 0 \\
1-\frac{1}{2} \mathrm{e}^{\xi} & \xi<0
\end{array} .\right.
$$

Note that in this example the travelling front is monotone on $\mathbb{R}$. However, this does not always have to be the case, particularly when choosing Mexican hat connectivities. For example with the choice $w(x)=$ $(1-a|x|) \mathrm{e}^{-|x|}$, we have that

$$
\psi(\xi)=\left\{\begin{array}{ll}
\mathrm{e}^{-\xi}(1-a-a \xi) & \xi \geq 0 \\
2(1-a)-\mathrm{e}^{\xi}(1-a+a \xi) & \xi<0
\end{array} .\right.
$$

To ensure that $\lim _{\xi \rightarrow-\infty} q(\xi)>h$, requires the choice $\int_{\mathbb{R}} w(y) \mathrm{d} y=2(1-a)>h$. Plots of (33) and (34) are shown in Fig. 1.

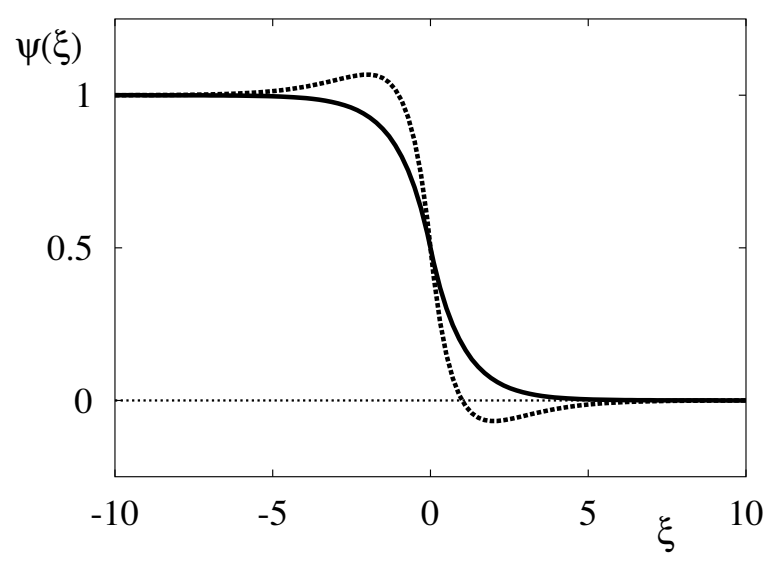

Figure 1: A plot of the travelling wave solution $\psi(\xi)$ for an exponential synaptic footprint $w(x)=\mathrm{e}^{-|x|} / 2$ (solid curve) and a wizard hat $w(x)=(1-|x| / 2) \mathrm{e}^{-|x|}$ (dashed curve). For both examples $\kappa=\int_{\mathbb{R}} w(y) \mathrm{d} y=$ $1 / 2$. Note that the wizard hat footprint leads to a nonmonotone shape for the travelling front.

\section{Recovery and lateral inhibition}

In real cortical tissues there are an abundance of metabolic processes whose combined effect is to modulate neuronal response. It is convenient to think of these processes in terms of feedback mechanisms that modulate synaptic currents. Such feedback may act to decrease activity in the wake of a travelling front so as to generate travelling pulses (rather than fronts). We will consider simple models of so-called spike frequency adaptation (i.e. the addition of a current that activates in the presence of high activity) that are known to lead to the generation of pulses for network connectivities that would otherwise only support travelling fronts [18]. Generalising the model in the previous 
section we write

$$
\begin{aligned}
Q u(x, t) & =(w \otimes f \circ u)(x, t)-g\left(w_{a} \otimes a\right)(x, t), \\
Q_{a} a(x, t) & =f \circ u(x, t),
\end{aligned}
$$

with $w_{a}(x)=w_{a}(|x|)$ and $g$ a constant. The (temporal) linear differential operator $Q_{a}$ is assumed to have a Green's function $\eta_{a}(t)$. With the alternative choice $Q_{a} a=u$ we obtain a model with linear recovery $[53,59]$. This model can also be analysed in exactly the same way as the model with nonlinear recovery, and we refer the reader to [51]. In [51] it is shown that stable coexisting travelling fronts can exist beyond a front bifurcation. Moreover, such fronts may be connected and depending on their relative speed the resulting region of activity can widen or contract.

The model with nonlinear recovery (35) may be written in integral form as

$$
u=\left[\eta * w \otimes-g \eta_{b} * w_{a} \otimes\right] f \circ u,
$$

where $\eta_{b}=\eta * \eta_{a}$. Note that we may also interpret this as a lateral inhibitory network model [60]. This model is expected to support travelling pulses of the form $q(\xi) \geq h$ for $\xi \in[0, \Delta]$ and $q(\xi)<h$ otherwise. We write this pulse solution in the form

$$
q(\xi)=\int_{0}^{\infty} \eta(z)\left[\psi(\xi+c z)-g \psi_{a}(\xi+c z)\right] \mathrm{d} z,
$$

where $\psi_{a}(\xi)=\int_{\mathbb{R}} w_{a}(y) a(\xi-y) \mathrm{d} y$. Here, $a(\xi)$ is given by

$$
a(\xi)= \begin{cases}\int_{-\xi / c}^{\Delta-\xi / c} \eta_{a}(z) \mathrm{d} z & \xi \leq 0 \\ \int_{0}^{\Delta-\xi / c} \eta_{a}(z) \mathrm{d} z & 0<\xi<\Delta, \\ 0 & \xi \geq \Delta\end{cases}
$$

and $\psi(\xi)$ by

$$
\psi(\xi)= \begin{cases}\mathcal{F}(-\xi, \Delta-\xi) & \xi \leq 0 \\ \mathcal{F}(0, \xi)+\mathcal{F}(0, \Delta-\xi) & 0<\xi<\Delta, \\ \mathcal{F}(\xi-\Delta, \xi) & \xi \geq \Delta\end{cases}
$$

where

$$
\mathcal{F}(a, b)=\int_{a}^{b} w(y) \mathrm{d} y .
$$

The dispersion relation $c=c(\Delta)$ is then implicitly defined by the simultaneous solution of $q(0)=h$ and $q(\Delta)=h$.
In a co-moving frame we have a modified form of (16) under the replacement $w(y) \eta(s) \rightarrow w(y) \eta(s)-$ $g w_{a}(y) \eta_{b}(s)$. Linearising around a travelling pulse solution and proceeding analogously as for the front solution described in section 5 , we obtain an eigenvalue equation of the form $u=\mathcal{L} u$. In this case

$$
\begin{aligned}
\mathcal{L} u(\xi) & =[A(\xi, \lambda)-g C(\xi, \lambda)] u(0) \\
& +[B(\xi, \lambda)-g D(\xi, \lambda)] u(\Delta),
\end{aligned}
$$

for $\xi \in[0, \Delta]$, with

$$
\begin{aligned}
A(\xi, \lambda) & =\frac{1}{c\left|q^{\prime}(0)\right|} \int_{\xi}^{\infty} \mathrm{d} y w(y) \eta(-\xi / c+y / c) \\
& \times \mathrm{e}^{-\lambda(y-\xi) / c}
\end{aligned}
$$

and $C(\xi, \lambda)$ is obtained under the replacement $w(y) \eta(s) \rightarrow w_{a}(y) \eta_{b}(s)$ in (42). Also $B(\xi, \lambda)=\left|q^{\prime}(0) / q^{\prime}(\Delta)\right| A(\xi-\Delta, \lambda)$ and $D(\xi, \lambda)=$ $\left|q^{\prime}(0) / q^{\prime}(\Delta)\right| C(\xi-\Delta, \lambda)$. Demanding that perturbations be determined self consistently at $\xi=0$ and $\xi=\Delta$ gives the system of equations

$$
\left[\begin{array}{l}
u(0) \\
u(\Delta)
\end{array}\right]=\mathcal{A}(\lambda)\left[\begin{array}{l}
u(0) \\
u(\Delta)
\end{array}\right]
$$

where

$$
\mathcal{A}(\lambda)=\left[\begin{array}{ll}
A(0, \lambda)-g C(0, \lambda) & B(0, \lambda)-g D(0, \lambda) \\
A(\Delta, \lambda)-g C(\Delta, \lambda) & B(\Delta, \lambda)-g D(\Delta, \lambda)
\end{array}\right] .
$$

There is a nontrivial solution of (44) if $\mathcal{E}(\lambda)=0$, where $\mathcal{E}(\lambda)=\operatorname{det}(\mathcal{A}(\lambda)-I)$. We interpret $\mathcal{E}(\lambda)$ as the Evans function of a travelling pulse solution of (36).

\subsection{Example: A pair of travelling pulses}

Here we consider the choice $\eta(t)=\alpha \mathrm{e}^{-\alpha t}, \eta_{a}(t)=\mathrm{e}^{-t}$, $w(x)=\mathrm{e}^{-|x|} / 2$ and $w_{a}=\delta(x)$ so that we recover a model recently discussed by Coombes et al. [34]. The travelling pulse solution for this model is given by (37) with $\psi_{a}(\xi)=a(\xi)$ and

$$
a(\xi)= \begin{cases}{\left[1-\mathrm{e}^{-\Delta / c}\right] \mathrm{e}^{\xi / c}} & \xi \leq 0 \\ {\left[1-\mathrm{e}^{(\xi-\Delta) / c}\right]} & 0<\xi<\Delta . \\ 0 & \xi \geq \Delta\end{cases}
$$


Using (39) $\psi(\xi)$ is given by

$$
\psi(\xi)= \begin{cases}\frac{1}{2}\left(\mathrm{e}^{m_{+} \xi}-\mathrm{e}^{m_{+}(\xi-\Delta)}\right) & \xi \leq 0 \\ 1-\frac{1}{2}\left(\mathrm{e}^{m_{+}(\xi-\Delta)}+\mathrm{e}^{m_{-} \xi}\right) & 0<\xi<\Delta . \\ \frac{1}{2}\left(\mathrm{e}^{m_{-}(\xi-\Delta)}-\mathrm{e}^{m_{-} \xi}\right) & \xi \geq \Delta\end{cases}
$$

In Fig. 2 we plot the speed of the pulse as a function of $g$, obtained by the simultaneous solution of $q(0)=h$ and $q(\Delta)=h$. It is straightforward to obtain $C(0, \lambda)=$

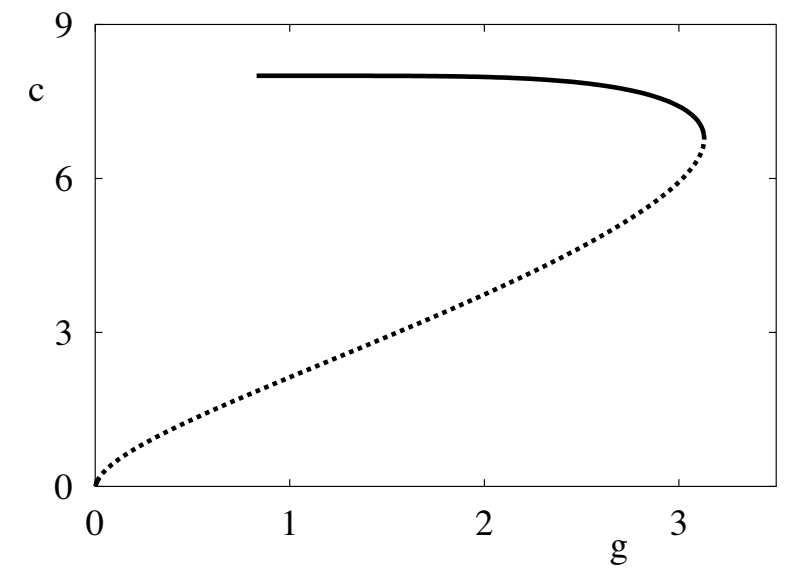

Figure 2: Speed of a travelling pulse as a function of $g$ in a model with nonlinear recovery. Parameters are $h=0.1$ and $\alpha=2$. The fast branch is stable (solid line), whilst the slow branch is unstable (dashed line).

$$
\begin{aligned}
& C(\Delta, \lambda)=D(\Delta, \lambda)=0 \text { and } \\
& A(0, \lambda)=\frac{1}{c\left|q^{\prime}(0)\right|} \frac{\alpha}{2} \frac{1}{1+\alpha / c+\lambda / c}, \\
& B(0, \lambda)=\frac{1}{c\left|q^{\prime}(\Delta)\right|} \frac{\alpha}{2}\left\{\frac{\mathrm{e}^{-(\alpha+\lambda) \Delta / c}-\mathrm{e}^{-\Delta}}{1-\alpha / c-\lambda / c}\right. \\
&\left.+\frac{\mathrm{e}^{-(\alpha+\lambda) \Delta / c}}{1+\alpha / c+\lambda / c}\right\}, \\
& D(0, \lambda)=\frac{\alpha \mathrm{e}^{-\Delta(1+\lambda) / c}}{c\left|q^{\prime}(\Delta)\right|}\left(\frac{1-\mathrm{e}^{-\Delta(\alpha-1) / c}}{\alpha-1}\right) .
\end{aligned}
$$

with $B(\Delta, \lambda)=\left|q^{\prime}(0) / q^{\prime}(\Delta)\right| A(0, \lambda)$ and $A(\Delta, \lambda)=$ $\mathrm{e}^{-\Delta} A(0, \lambda)$. Moreover, we have simply that $-c q^{\prime}(\phi) / \alpha=-h+\psi(\phi)-g a(\phi)$ for $\phi \in\{0, \Delta\}$. One natural way to find the zeros of $\mathcal{E}(\lambda)$ is to write $\lambda=\nu+\mathrm{i} \omega$ and plot the zero contours of $\operatorname{Re} \mathcal{E}(\lambda)$ and $\operatorname{Im} \mathcal{E}(\lambda)$ in the $(\nu, \omega)$ plane. The Evans function is zero where the lines intersect. We do precisely this in Fig. 3 for three distinct points on the solution branch shown in Fig. 2. On the fast branch it would appear that all the zeros of the Evans function lie in the left hand complex plane, whilst for the slow wave there is at least one in the right hand plane (on the real axis). As expected there is a double zero eigenvalue as one passes from the fast to the slow branch of travelling pulse solutions. Hence, the fast wave is stable and the slow wave unstable.

\subsection{Example: A dynamic instability of a standing pulse}

In many models of working memory, transient stimuli are encoded by feature-selective persistent neural activity. Such stimuli are imagined to induce the formation of a spatially localised bump of persistent activity which coexists with a stable uniform state (with low firing rate). Although long-range inhibition with local recurrent excitation [12, 61] (and indeed without [62]) is known to be one mechanism for bump formation, it is also possible that bistability at the single neuron level may have a role to play [63]. Here, we shall focus on the former mechanism and refer the reader to Fall et al. [64] for a recent discussion of this phenomenon within the Camperi-Wang population model of visuospatial working memory with purely lateral inhibition [63].

Localised bump solutions are easily investigated within the framework that we have described here as they may be regarded as standing pulses with $c=0$. In this case (37) reduces to

$$
q(\xi)=\int_{0}^{\Delta} w_{b}(\xi-y) \mathrm{d} y,
$$

where we have introduced the effective interaction kernel $w_{b}(x)=w(x)-g w_{a}(x)$. To be more specific (50) describes a so-called 1-bump solution. Although it is possible to pursue a similar construction for 2-bump solutions, these are typically found to be unstable for a Heaviside firing rate function [13], though this is not necessarily true when one considers smooth firing rate functions [34]. From (50) we may calculate the derivative

$$
q^{\prime}(\xi)=w_{b}(\xi)-w_{b}(\xi-\Delta)
$$



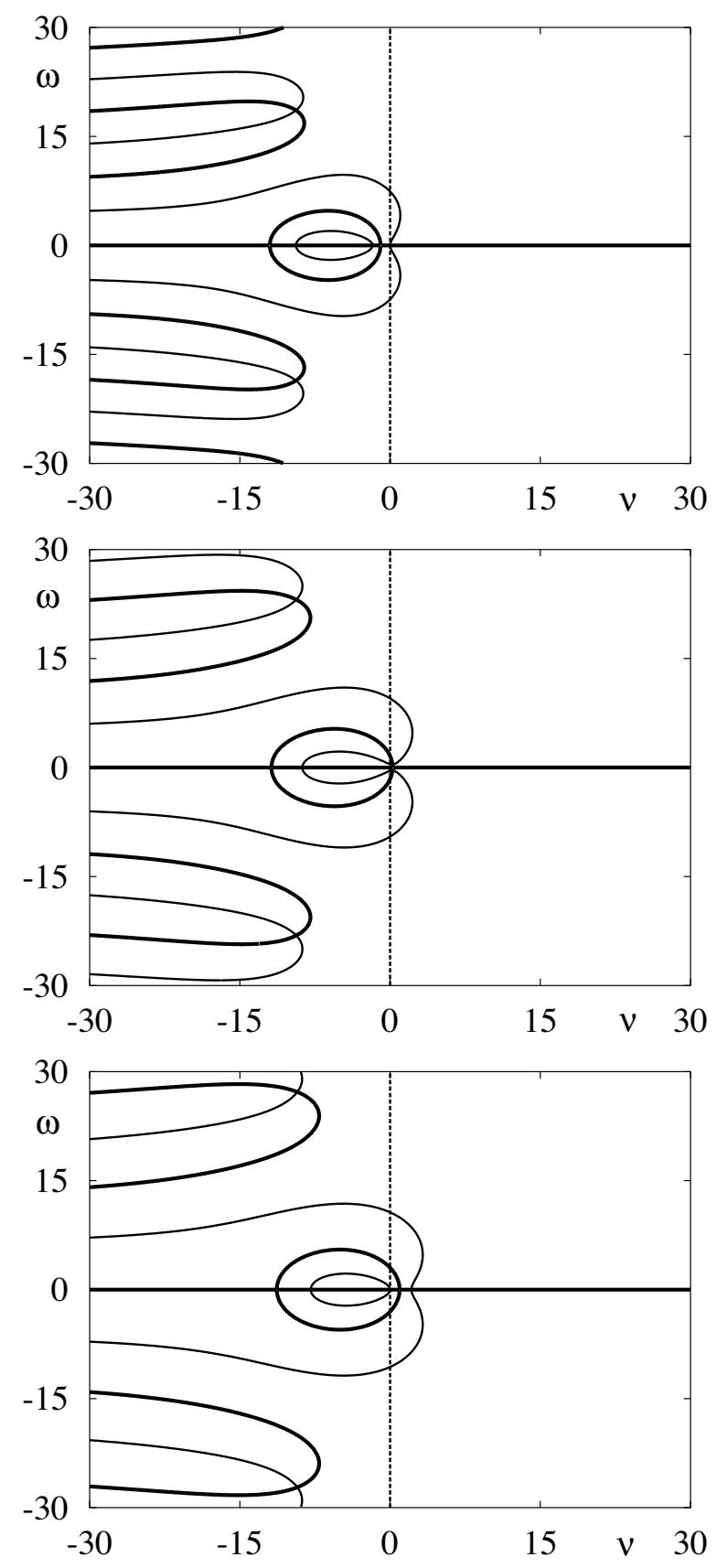

Figure 3: Evans function for a travelling pulse in a model with nonlinear recovery. Zeros of the Evans function occur at the intersection of the thick and thin solid lines where $\operatorname{Re} \mathcal{E}(\lambda)=0=\operatorname{Im} \mathcal{E}(\lambda)$. In the top figure $g=3$ and a solution is taken from the fast branch. In the middle the value of $g$ is that at the saddle-node bifurcation from Fig. 2. On the bottom $g=3$ with a solution taken from the slow branch. Other parameters are the same as in Fig. 2. from which we note that $\left|q^{\prime}(0)\right|=\left|q^{\prime}(\Delta)\right|$. For $c=0$, $w(y)$ and $w_{a}(y)$ are relatively flat and we obtain the further simplification

$$
\begin{aligned}
& A(\xi, \lambda)=\frac{1}{\left|q^{\prime}(0)\right|} \operatorname{LT}[\eta](\lambda) w(\xi), \\
& C(\xi, \lambda)=\frac{1}{\left|q^{\prime}(0)\right|} \operatorname{LT}\left[\eta_{b}\right](\lambda) w_{a}(\xi),
\end{aligned}
$$

with $B(\xi, \lambda)=A(\Delta-\xi, \lambda)$ and $D(\xi, \lambda)=C(\Delta-\xi, \lambda)$. In this section we choose $\eta(t)=\alpha \mathrm{e}^{-\alpha t}, \eta_{a}(t)=\mathrm{e}^{-t}$, $w(x)=\mathrm{e}^{-|x|} / 2$ and $w_{a}(x)=\mathrm{e}^{-|x| / \sigma_{a}} /\left(2 \sigma_{a}\right)$. Enforcing the condition $q(0)=h$ or $q(\Delta)=h$ generates the pulse width as a function of system parameters:

$$
\frac{1}{2}\left(1-\mathrm{e}^{-\Delta}\right)-\frac{g}{2}\left(1-\mathrm{e}^{-\Delta / \sigma_{a}}\right)=h .
$$

A plot of the pulse width as a function of the threshold parameter $h$ is shown in Fig. 4, highlighting the fact that solutions come in pairs. The standing pulse shape

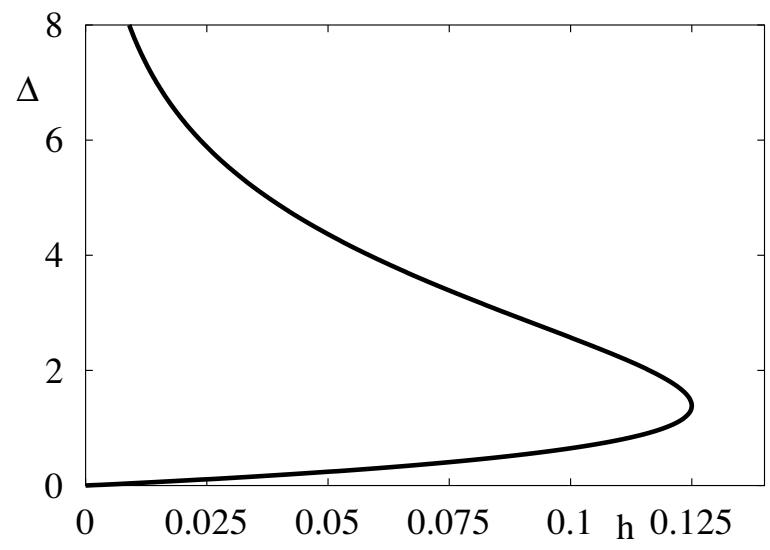

Figure 4: Pulse width as a function of threshold $h$ in a model with lateral inhibition and nonlinear recovery. Here $\sigma_{a}=2$ and $g=1$.

is easily calculated and some typical bump shapes are shown in Fig. 5. For small values of the threshold the bump on the upper branch of Fig. 4 has a dimpled shape such that $q^{\prime \prime}(0)>0$. With increasing $h, q^{\prime \prime}(0)$ decreases through zero and a bump with $q^{\prime \prime}(0)<0$ is obtained.

We may use (52) and (53) to construct the Evans function and plot it in the same fashion as the last example. However, unlike the last example we find that there is not a simple exchange of stability as one 

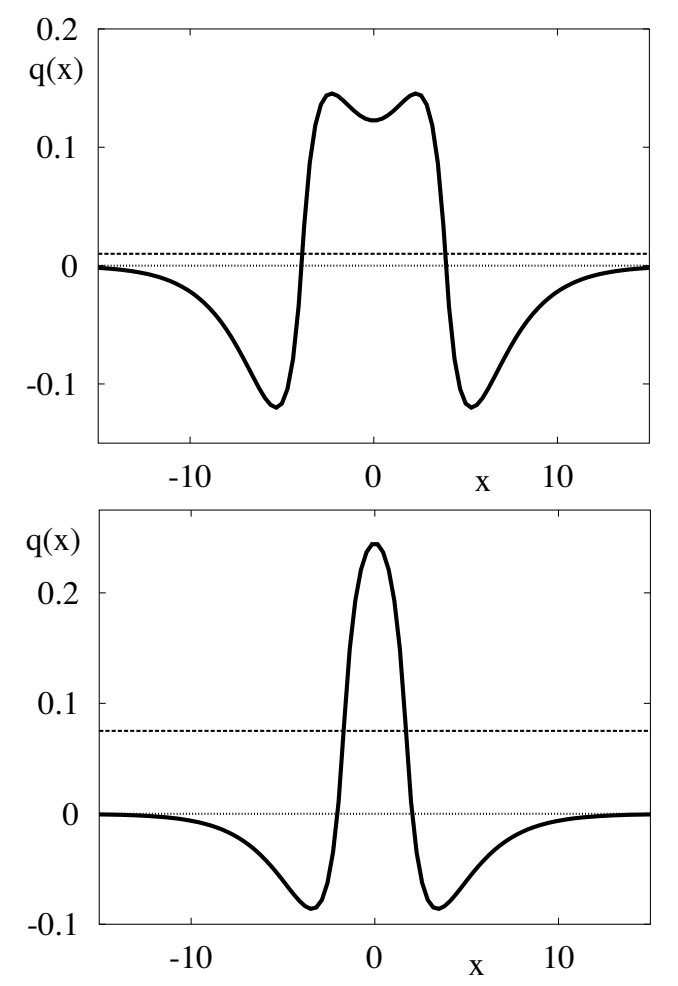

Figure 5: Bumps in a model with lateral inhibition and nonlinear recovery, with $\sigma_{a}=2$ and $g=1$. Top: $(h, \Delta)=(0.01,7.78)$. Bottom: $(h, \Delta)=(0.075,3.39)$. Both solutions are taken from the upper branch of Fig. 4, showing that dimple solutions are found for a sufficiently small choice of threshold.

passes through the limit point defining the transition from a broad to a narrow pulse. Indeed we see from Fig. 6 that it is possible for a solution on the upper branch of Fig. 5 to undergo a dynamic instability with increasing $\alpha$. By dynamic we mean that a pair of complex eigenvalues crosses into the right hand plane on the imaginary axis, so that the standing pulse may begin to oscillate, as originally described in [60]. For the parameter values in Fig. 6 and choosing a value of $\alpha$ below that defining a dynamic instability, direct numerical simulations show that a bump solution is stable to random perturbations. In contrast, beyond the dynamic instability point, a bump solution can destabilise in favour of a homogeneous steady state, as illustrated in Fig. 7.

To gain more insight into the form of a solution beyond the oscillatory instability it is useful to assume a
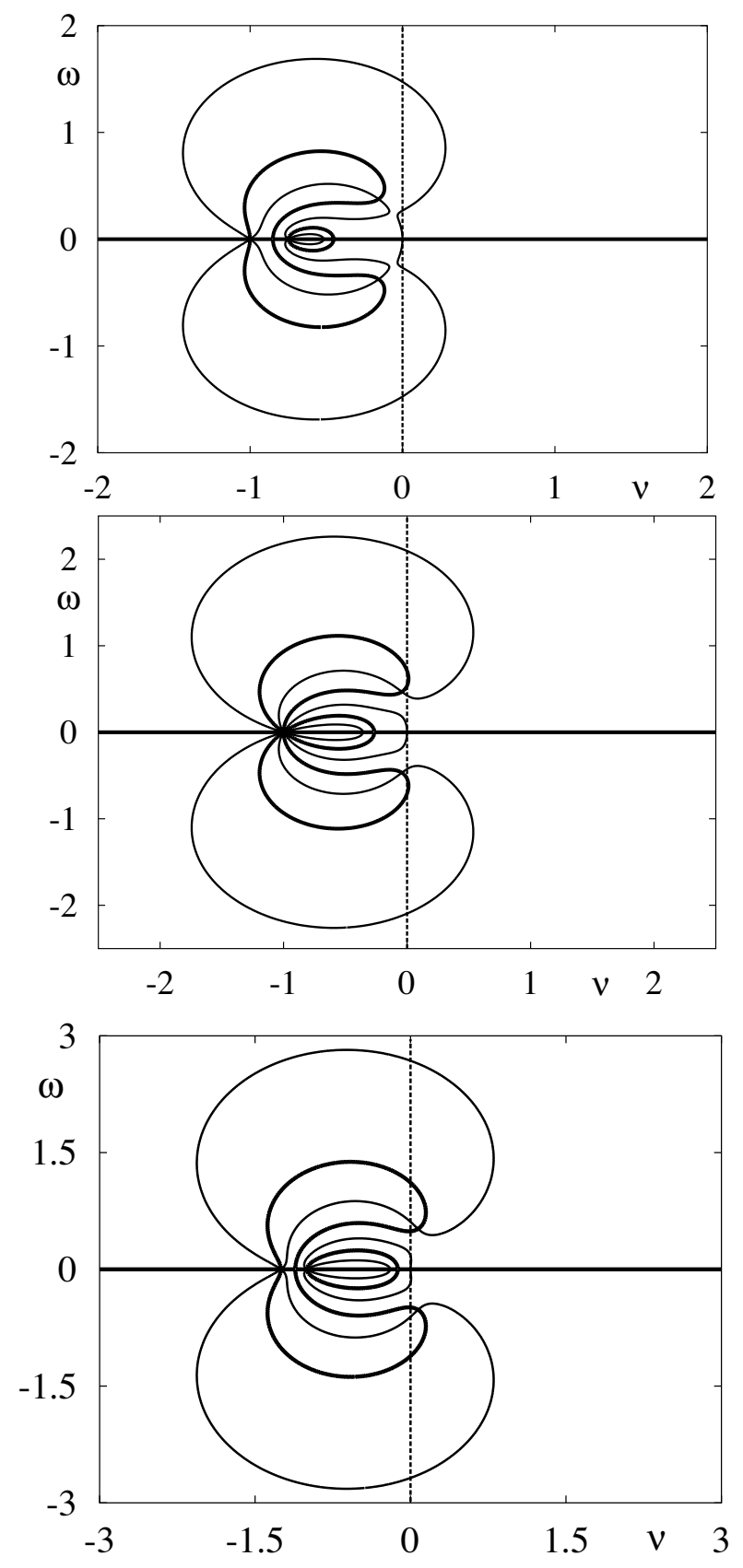

Figure 6: Evans function for a bump in the model with lateral inhibition and nonlinear recovery. Here $h=0.1$ and a solution is taken from the branch with largest width $\Delta$. On the top $\alpha=0.75$, and in the middle $\alpha=$ 1.0, whilst on the bottom $\alpha=1.25$. This illustrates the possibility of a dynamic instability with increasing $\alpha$ as a pair of complex eigenvalues crosses over to the right hand plane through the imaginary axis. 


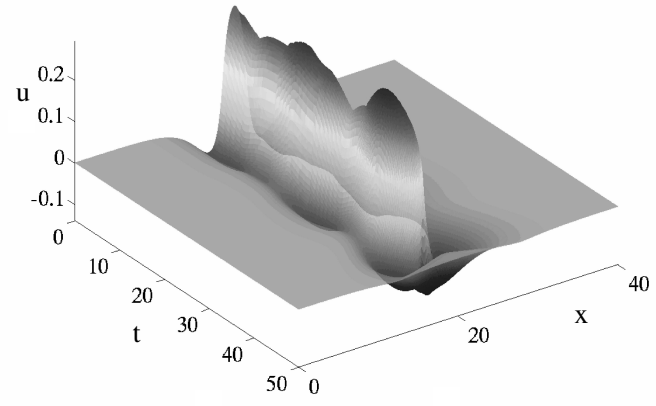

Figure 7: 3-d plot of a destabilised bump $(\alpha=1.1)$, with $h=0.1$. Initial data with $u(x, 0)=1.05 q(x)$ where $q(x)$ is the stationary bump solution.

solution of the form $u\left(x_{i}(t), t\right)=h$ for $i=1,2$, such that $x_{1}<x_{2}$ and $u(x, t)>h$ for $x \in\left(x_{1}(t), x_{2}(t)\right)$ at time $t$ and $u(x, t)<h$ otherwise. Differentiation of this defining expression for a time-dependent bump gives

$$
u_{x}\left(x_{i}, t\right) \frac{\mathrm{d} x_{i}}{\mathrm{~d} t}+u_{t}\left(x_{i}, t\right)=0 .
$$

This equation can be used to obtain the evolution of the bump-width $\Delta(t)=x_{2}(t)-x_{1}(t)$. The expressions for $u_{t}$ and $u_{x}$ are naturally obtained by differentiating (36) (remembering that $f$ is a Heaviside). For example, if we choose $Q=1+\alpha^{-1} \partial_{t}, Q_{a}=1+\partial_{t}$, then the bump evolves according to

$$
\begin{aligned}
\frac{y}{2} \frac{\mathrm{d} \Delta}{\mathrm{d} t} & =\alpha(-h+W(\Delta)-z), \\
\frac{\mathrm{d} z}{\mathrm{~d} t} & =-z+g W_{a}(\Delta), \\
\frac{\mathrm{d} y}{\mathrm{~d} t} & =\alpha\left(-y+w(0)-w(\Delta)-g z_{1}\right), \\
\frac{\mathrm{d} z_{1}}{\mathrm{~d} t} & =-z_{1}+w_{a}(0)-w_{a}(\Delta),
\end{aligned}
$$

where we identify $y=u_{x}\left(x_{1}, t\right)=-u_{x}\left(x_{2}, t\right)$. Here

$$
\begin{gathered}
W(x)=\int_{0}^{x} w(y) \mathrm{d} y=\frac{1}{2}\left[1-\mathrm{e}^{-x}\right], \\
W_{a}(x)=\int_{0}^{x} w_{a}(y) \mathrm{d} y=\frac{1}{2}\left[1-\mathrm{e}^{-x / \sigma_{a}}\right] .
\end{gathered}
$$

Denoting the fixed point by $\left(\Delta^{*}, z^{*}, y^{*}, z_{1}^{*}\right)$, we recover an expression for $\Delta^{*}$ identical to earlier, i.e. $W\left(\Delta^{*}\right)-$ $g W_{a}\left(\Delta^{*}\right)=h$, which is equivalent to (54). Hence, the curve for $\Delta^{*}=\Delta^{*}(h)$ is identical to that of Fig. 4. The Jacobian of the system at the fixed point has eigenvalues $\lambda=-1, \lambda=-\alpha$ and $\lambda=\lambda_{ \pm}$where

$$
\lambda_{ \pm}=\frac{\operatorname{Tr} J \pm \sqrt{(\operatorname{Tr} J)^{2}-4 \operatorname{det} J}}{2},
$$

and

$$
J=\left[\begin{array}{cc}
2 \alpha w\left(\Delta^{*}\right) / y^{*} & -2 \alpha / y^{*} \\
g w_{a}\left(\Delta^{*}\right) & -1
\end{array}\right] .
$$

We note that $\operatorname{det} J=-2 \alpha w_{b}\left(\Delta^{*}\right) / y^{*}$. Moreover, differentiation of the fixed point equation for $\Delta^{*}$ with respect to $\Delta^{*}$ yields $w_{b}\left(\Delta^{*}\right)=h^{\prime}\left(\Delta^{*}\right)$, showing that $w_{b}\left(\Delta^{*}\right)>0$ on the lower solution branch of Fig. 4 . Hence, $\operatorname{det} J<0$, and from (60), we see that this solution is a saddle. On the upper branch $\operatorname{det} J>0$ and a Hopf bifurcation occurs when $\operatorname{Tr} J=0$, which is expected to occur with increasing $\alpha$. A plot of the fixed point of the system of equations (56) as well as the maximum amplitude of oscillation for periodic orbits arising at a Hopf bifurcation are plotted in Fig. 8 . This numerical solution of (56) shows that the Hopf

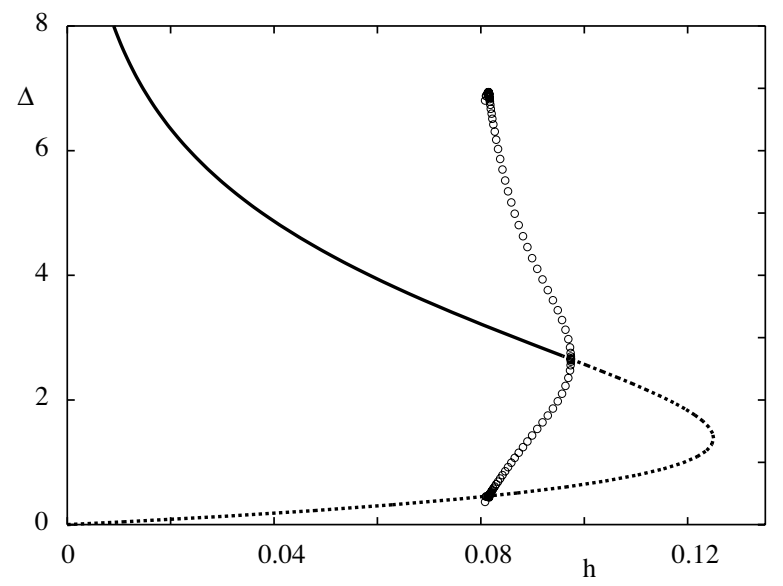

Figure 8: Pulse width as a function of threshold $h$ in a model with lateral inhibition and nonlinear recovery. Here $\sigma_{a}=2, g=1$ and $\alpha=4$. Solid (dashed) lines are stable (unstable). Open circles denote the maximum amplitude of unstable periodic orbits emerging from a sub-critical Hopf bifurcation.

bifurcation is sub-critical, with no emerging stable orbits. In fact the emergent unstable periodic orbit is destroyed in a collision with the unstable lower branch of fixed points. Pinto and Ermentrout have suggested 
that this is the reason why direct numerical simulations (just beyond the point of instability) do not show stable breathing bumps [60]. Although this approach is useful in predicting qualitative behaviour of the full equations of motion, it is not particularly useful in providing accurate estimates of the critical values of $\alpha$ and $h$ necessary to see a dynamical instability. Unfortunately, there is not an accurate agreement with the point of instability calculated using the exact Evans function approach and that of the Hopf bifurcation in the kinematic theory of bump dynamics.

Returning our attention to the results described above for dynamic instabilities of localised bumps, it would appear that the question of how to generate stable breathing solutions in a neural field model is an interesting one. One way to generate such solutions has been found that relies upon the inclusion of localised inputs [65], breaking the homogeneous structure of the network. The use of unimodal inputs means that this mechanism does not require a Mexican hat connectivity to either generate bumps or stable breathing bumps (merely just a positive footprint such as a spatially decaying exponential). However, it is also possible to find stable breathing solutions in a homogeneous model with Mexican hat connectivity that incorporates a dynamic firing threshold [66].

\section{Space-dependent delays}

In the presence of space-dependent delays, it is natural for $\psi(x, t)$ to take the slightly more general form

$$
\psi(x, t)=\int_{-\infty}^{\infty} \int_{-\infty}^{\infty} K(x-y, t-s) f \circ u(y, s) \mathrm{d} y \mathrm{~d} s
$$

A model with space-dependent axonal delays may be obtained by choosing $K(x, t)=w(x) \delta(t-|x| / v)$ $[34,67]$, where $v$ is the finite speed of action potential propagation. Alternatively a model of dendritic delays studied intensively by Bressloff $[68,69]$ is recovered with the choice $K(x, t)=w(x) g(t)$. Here $g(t)$ is the Green's function of the cable equation with a synapse at a fixed (dendritic) distance from the cell body. As in section 2 the (double) convolution structure of this equation may be exploited to obtain a PDE formula- tion, provided that the two dimensional FT of $K(x, t)$ has a rational structure. For an axonal delay the choice $w(x)=\mathrm{e}^{-|x|} / 2$ gives rise to a type of damped inhomogeneous wave equation:

$$
\left[\left(v+\partial_{t}\right)^{2}-v^{2} \partial_{x x}\right] \psi(x, t)=\left[v^{2}+v \partial_{t}\right] f \circ u(x, t) .
$$

This equation was first derived by Jirsa and Haken $[67,70]$ and has been studied intensively in respect to the brain-behaviour experiments of Kelso et al. [71]. Similar equations have been presented in $[72,73,74$, 75], where the linearisation of such equations (about a homogeneous steady state) has been used in the interpretation of EEG spectra. As regards the set of full nonlinear integral equations one obvious consequence of introducing an axonal delay is that the speed of a travelling wave must be slower than that of the action potential, i.e. $c<v$. The calculation of wave speed and stability for a Heaviside firing rate function is easily generalised to the case of finite $v$ and is described in $[18,34,51]$. For the case of the exponential synaptic footprint chosen above and an exponential synaptic response, $\eta(t)=\alpha \mathrm{e}^{-\alpha t}$, it is possible to obtain a closed form expression for the speed, $c$, of a front in terms of the speed of an action potential, $v$, as

$$
c=\frac{v(2 h-1)}{2 h-1-2 h v / \alpha} \text {. }
$$

Note that we recover equation (31) in the limit $v \rightarrow \infty$ as expected. The techniques used in section 6 may also be adapted to construct travelling pulse solutions, and indeed this has recently been done by Enculescu [76]. This calculation is easily reproduced (although we do not do so here) and we have used this to make a plot of front and pulse speed as a function of $v$ in Fig. 9. We note that the pulse (if it exists) always travels slower than the front. Interestingly an examination of the Evans function for each solution (adapting the calculations in sections 5 and 6) shows that the front is always stable whilst the pulse is always unstable. Hence, it is not possible to change the stability of a front or pulse by varying $v$. However, the affect of varying $v$ can have a far more profound effect on the stability of a homogeneous steady state, as we now discuss. 


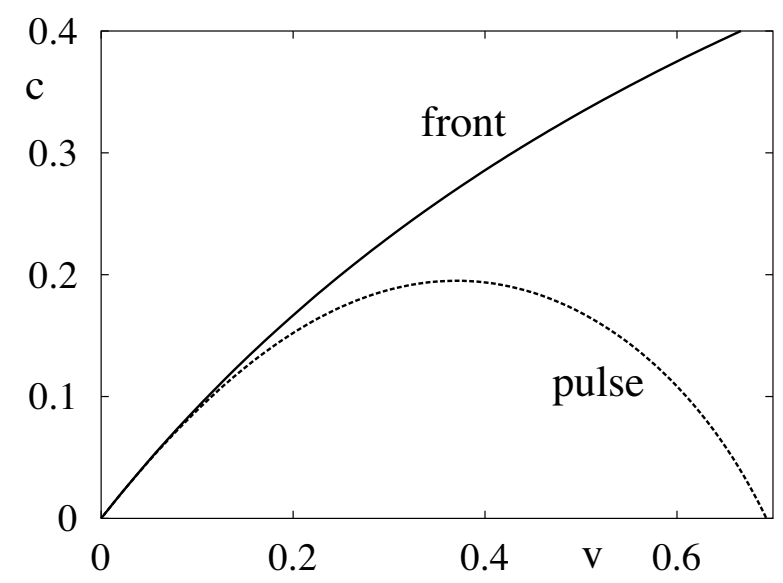

Figure 9: The speed of a travelling front (solid line) and a travelling pulse (dashed line) as a function of action potential velocity in a model with space dependent axonal delays. Here the synaptic footprint is exponential, $w(x)=\mathrm{e}^{-|x|} / 2$, and the synaptic response function is also exponential, $\eta(t)=\alpha \mathrm{e}^{-\alpha t}$. The firing rate function is a Heaviside, $f(u)=\Theta(u-h)$. Note that the pulse travels slower than the front. Moreover, an examination of the Evans function for both solution types shows that the front is always stable, and the pulse is always unstable. Parameters are $\alpha=1$ and $h=0.25$.

In section 3, we showed that static Turing instabilities can arise for Mexican hat connectivities in the absence of space-dependent delays. However, when working with (62) it is possible for dynamic Turing instabilities to occur. These were first found in neural field models by Bressloff [68] for dendritic delays and more recently by Hutt et al. [77] for axonal delays. Both these studies suggest that a combination of inverted Mexican hat connectivity with a space-dependent delay may lead to a dynamic instability. Indeed the choice of inverted Mexican hat is natural when considering cortical tissue and remembering that principal pyramidal cells i) are often enveloped by a cloud of inhibitory interneurons, and ii) that long range cortical connections are typically excitatory. We now illustrate the possibility of a dynamic Turing instability for a model with axonal delays by considering an inverted wizard hat function $w(x)=(|x|-1) \mathrm{e}^{-|x|}$ and alpha function synaptic response $\eta(t)=\alpha^{2} t \mathrm{e}^{-\alpha t}$. Proceeding as in section 3 we linearise around a homogeneous solution and consider perturbations of the form $\mathrm{e}^{\lambda t} \mathrm{e}^{i k x}$. In this case the dispersion relation for $\lambda=\lambda(k)$ takes the modified form

$$
\gamma \operatorname{LT}[\eta](\lambda) \operatorname{FT}[\widehat{w}](k)-1=0,
$$

where $\widehat{w}(x)=w(x) \mathrm{e}^{-\lambda|x| / v}$. Compared to (13) (obtained in the absence of space-dependent delays), equation (65) is not separable in the sense that $\operatorname{FT}[\widehat{w}](k)$ is not just a function of $k$, but is also a function of $\lambda$. It is natural to decompose $\lambda$ in the form $\lambda=\nu+i \omega$ and equate real and imaginary parts of (65) to obtain two equations for $\nu$ and $\omega$. If we write these in the form $G(\nu, \omega)=0$ and $H(\nu, \omega)=0$, then the simultaneous solution of these two equations gives the pair $(\nu(k), \omega(k))$, so that we may parametrically express $\omega=\omega(\nu)$. An example of such a plot is shown in Fig. 10. Here, it can be seen that for a fixed value

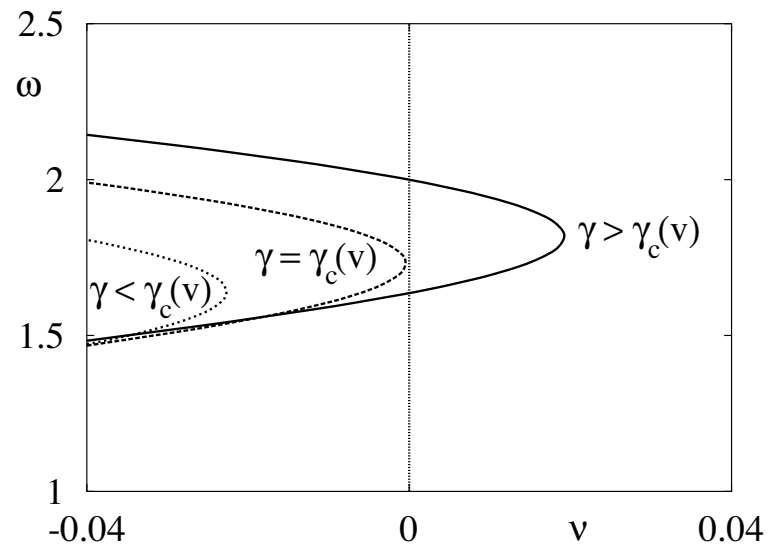

Figure 10: Continuous spectrum for a scalar neural field model with an inverted wizard hat synaptic footprint, axonal delays (of speed $v=1$ ) and an alpha function synapse (with $\alpha=1$ ). On the left $\gamma=7<$ $\gamma_{c}(v)$ and on the right $\gamma=9>\gamma_{c}(v)$. For $\gamma<\gamma_{c}(v)$ the continuous spectrum lies in the left hand complex plane and the homogeneous solution is stable. For $\gamma>\gamma_{c}(v)$ part of the continuous spectrum lies in the right hand complex plane and the homogeneous solution is unstable.

of $v$ there is a critical value of $\gamma=\gamma_{c}(v)$ such that for $\gamma<\gamma_{c}(v)$, the continuous spectrum lies in the left hand 
complex plane, whilst for $\gamma>\gamma_{c}(v)$ part of the spectrum lies in the right hand complex plane. The Turing bifurcation point is defined by the smallest nonzero wave number $k_{c}$ that satisfies $\operatorname{Re}\left(\lambda\left(k_{c}\right)\right)=0$. It is said to be static if $\operatorname{Im}\left(\lambda\left(k_{c}\right)\right)=0$ and dynamic if $\operatorname{Im}\left(\lambda\left(k_{c}\right)\right) \equiv \omega_{c} \neq 0$. A static bifurcation may then be identified with the tangential intersection of $\omega=\omega(\nu)$ and $\nu=0$ at $\omega=0$. Similarly a dynamic bifurcation is identified with a tangential intersection with $\omega \neq 0$. The integral transforms in (65) are easily calculated as $\operatorname{LT}[\eta](\lambda)=(1+\lambda / \alpha)^{-2}$ and

$$
\widehat{w}(k, \lambda)=\frac{-2 \lambda / v\left[(1+\lambda / v)^{2}-k^{2}\right]-4 k^{2}(1+\lambda / v)}{\left[(1+\lambda / v)^{2}+k^{2}\right]^{2}},
$$

so that we may rewrite (65) as a sixth order polynomial in $\lambda ; \sum_{n=0}^{6} a_{n} \lambda^{n}=0$ where the coefficients $a_{n}=$ $a_{n}(k, v, \alpha, \gamma)$ are given in the appendix. Hence, the functions $G(\nu, \omega)$ and $H(\nu, \omega)$ may also be written as polynomials in $(\nu, \omega)$. For the calculation of a dynamic Turing instability we are required to track points in parameter space for which $\nu^{\prime}(\omega)=0$. By differentiating $G(\nu, \omega)=0=H(\nu, \omega)$ with respect to $\omega$ we see that this is equivalent to tracking points where $G_{k} H_{\omega}-H_{k} G_{\omega}=0$ (itself another polynomial equation). Beyond a dynamic Turing instability we expect the growth of travelling patterns of the form $\mathrm{e}^{i\left(\omega_{c} t+k_{c} x\right)}$. A plot of the critical curve $\gamma=\gamma_{c}(v)$ for a dynamic Turing instability (with $k_{c} \neq 0$ ) is shown in Fig. 11. Here, it can be seen that with increasing $\gamma$ (the gradient of the firing rate at the homogeneous steady state) a dynamic instability is first met for $v \sim 1$. Direct numerical simulations (not shown) of the full model show excellent agreement with the predictions of the dynamic Turing instability analysis. To determine the conditions under which one might see a standing wave (arising from the interaction of a left and right travelling wave), it is necessary to go beyond linear analysis and determine the evolution of mode amplitudes. The techniques to do this in (one dimensional) neural field theories are nicely described by Curtu and Ermentrout [78].

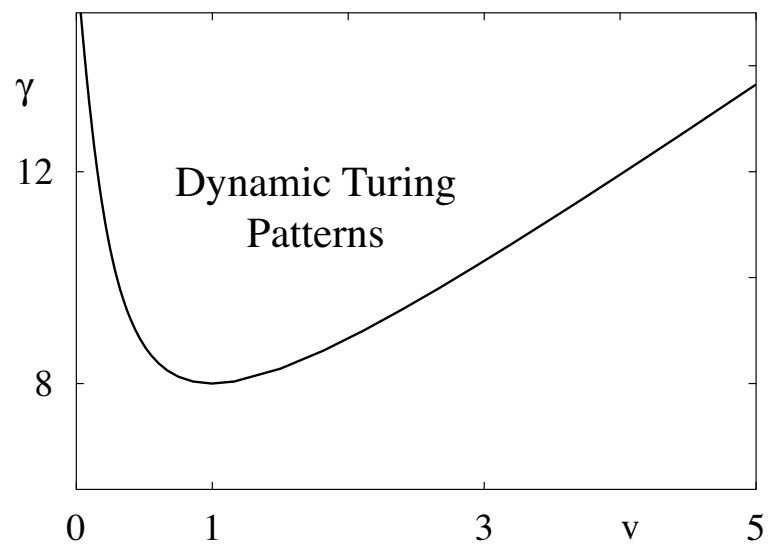

Figure 11: The critical curve for a dynamic Turing instability in a neural field with axonal delays and an inverted wizard-hat connectivity, with an alpha function synaptic response (and $\alpha=1$ ). Above the curve $\gamma=\gamma_{c}(v)$, the homogeneous steady state is unstable, leading to the growth of travelling patterns of the form $\mathrm{e}^{i\left(\omega_{c} t+k_{c} x\right)}$.

\section{Neural field equations with slow ionic currents}

In the type of continuum models we have considered so far it has been assumed that the synaptic input current is a function of the pre-synaptic firing rate function. To see how this might arise consider a one dimensional continuum of spiking single neurons with synaptic input at position $x$ given by

$$
u(x, t)=\eta * w \otimes \sum_{m \in \mathbb{Z}} \delta\left(t-T^{m}(x)\right) .
$$

This models the effect of an idealised action potential (delta-Dirac function) arriving at a synapse and initiating a postsynaptic current at time $T^{m}$. If the synaptic response is on a slower time scale than that of the mean interspike-interval $\left(T^{m}-T^{m-1}\right)$ and fluctuations around the mean are small, then it is natural to replace the spike train in (67) with a (smooth) firing rate function (see for example $[79,80]$ ). To illustrate how one might go about deriving this firing rate function we consider an integrate-and-fire process for the evolution of a cell membrane voltage given by

$$
C \frac{\partial v}{\partial t}=-g_{L}\left(v-v_{L}\right)+\sum_{k=1}^{N} I_{k}+u .
$$


Here, $C$ is a membrane capacitance, $g_{L}$ a constant leakage conductance, and $v_{L}$ a constant leakage reversal potential. The $I_{k}$ represent a set of slow intrinsic ionic currents, which typically have the form $I_{k}=g_{k} m_{k}^{p_{k}} h_{k}^{q_{k}}\left(v_{k}-v\right)$ where $p_{k}, q_{k} \in \mathbb{Z}, m_{k}$ and $h_{k}$ are gating variables that satisfy differential equations, $v_{k}$ is the reversal potential of the $k$ th channel and the $g_{k}$ are a set of constant conductances. From now on we shall refer all voltages to $v_{L}$. An action potential is said to occur whenever $v$ reaches some threshold $h$. The set of action potential firing times are defined by

$$
T^{m}(x)=\inf \left\{t \mid v(x, t) \geq h ; t \geq T^{m-1}(x)+\tau_{R}\right\} .
$$

Here $\tau_{R}$ represents an absolute refractory period. Immediately after a firing event the system undergoes a discontinuous reset such that $v \rightarrow 0$. Assuming that the dynamics for $v$ is much faster than that of $u$ and any intrinsic currents (equivalent to taking $\tau \equiv C / g_{L} \rightarrow 0$ in (68)), then $v$ equilibrates to it's steady-state value, which we denote by $v_{\mathrm{ss}}=$ $v_{\mathrm{ss}}\left(u, m_{1}, \ldots, m_{N}, h_{1}, \ldots, h_{N}\right)$. Moreover, we may compute the firing rate of the IF process as $f=f\left(v_{\mathrm{ss}}\right)$, where

$$
f(v)=\frac{1}{\tau_{R}+\tau \ln (v /(v-h))} \Theta(v-h), \quad \tau=\frac{C}{g_{L}} .
$$

So a neural field model that respects the presence of intrinsic ionic currents should be written as

$$
u=\eta * w \otimes f \circ v_{s s} .
$$

Note that in the absence of any slow intrinsic currents we obtain the standard model $u=\eta * w \otimes f \circ u$, since $v_{\mathrm{ss}}=u$ (after choosing units such that $\left.g_{L}=1\right)$.

To demonstrate the enormous impact the inclusion of extra slow currents can have, consider a single intrinsic ionic current $\left(N=1\right.$ in (68)) with $p_{1}=q_{1}=1$, $m_{1}=\Theta\left(v_{\mathrm{ss}}-v_{r}\right)$ and $h_{1}=r$ given by

$$
\tau\left(v_{\mathrm{ss}}\right) \frac{\mathrm{d} r}{\mathrm{~d} t}=-r+r_{\infty}\left(v_{\mathrm{ss}}\right),
$$

with $r_{\infty}(v)=\Theta\left(v_{r}-v\right)$ and $\tau(v)=\tau^{-} \Theta\left(v-v_{r}\right)+$ $\tau^{+} \Theta\left(v_{r}-v\right)$. This is a minimal model for the slow T-type calcium current $I_{T}$ [81]. The slow variable $r$ represents the deinactivation of the low-threshold $\mathrm{Ca}^{2+}$ conductance. When this conductance is evoked,
$\mathrm{Ca}^{2+}$ entering the neuron via T-type $\mathrm{Ca}^{2+}$ channels causes a large voltage depolarisation known as the low-threshold $\mathrm{Ca}^{2+}$ spike (LTS). Conventional action potentials mediated by fast $\mathrm{Na}^{+}$and $\mathrm{K}^{+}$currents often ride on the crest of an LTS resulting in a burst response (i.e., a tight cluster of spikes). If the neuron is hyperpolarised below $v_{r}$, the low-threshold current deinactivates (with a time constant of $\tau^{-}$). In this situation release from inhibition results in a post inhibitory rebound response consisting of an LTS and a cluster of 210 spikes. This type of dynamical behaviour is known to play an important role within the context of thalamocortical oscillations [25]. When neurons can fire via post inhibitory rebound it is also well known that this can lead to lurching waves of activity propagating through an inhibitory network [82]. A lurching wave does not travel with a constant profile, (i.e., there is no travelling wave frame) although it is possible to identify a lurching speed. Rather, the propagating wave recruits groups of cells in discrete steps. The leading edge of active cells inhibits some cluster of cells ahead of it (depending on the size of the synaptic footprint). Inhibited cells (ahead of the wave) must wait until they are released from inhibition before they can, in turn, fire. The first mathematical analysis of this phenomenon can be attributed to Terman et al. [83]. These authors work with a slightly more complicated version of the slow $I_{T}$ current than considered here and treat conductance based models (rather than firing rate). Using techniques from geometric singular perturbation theory they derive explicit formulas for when smooth and lurching waves exist and also determine the effect of network parameters on wave speed. However, this work relies partly on numerically determined properties of the single cell model.

Here we show how an exact analysis of lurching waves can be performed when the $I_{T}$ current described above is incorporated into a firing rate model. Taking $v_{1} \gg v$ and introducing $g_{r}=g_{1} v_{1}$, then $v_{\mathrm{ss}}=$ $v_{\mathrm{ss}}(u, r)$ is given by the solution to

$$
v_{\mathrm{ss}}=u+g_{r} r \Theta\left(v_{\mathrm{ss}}-v_{r}\right),
$$

choosing units such that $g_{L}=1$. For mathematical convenience we work with the Heaviside firing rate 
function $f(v)=\Theta(v-h)$ (obtained from (70) in the limit $\tau \rightarrow 0$ with units such that $\tau_{R}=1$ ) and consider a purely inhibitory network with

$$
w(x)=-\frac{1}{2 \sigma} \Theta(\sigma-|x|) .
$$

We denote the size of a cluster involved in a lurch by $L$. For simplicity we shall only consider lurching pulses

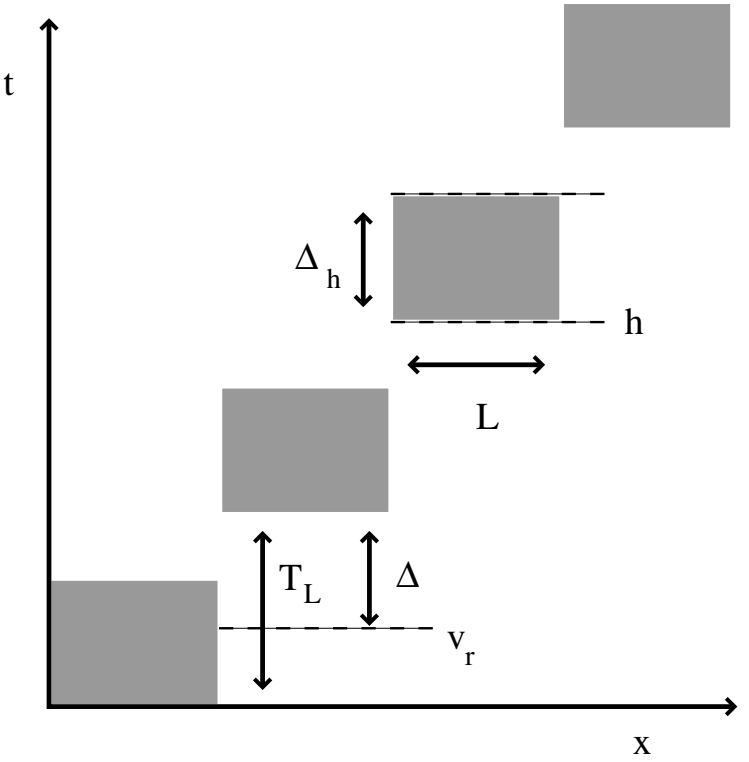

Figure 12: A diagram of an idealised solitary lurching pulse showing the four unknowns that parameterise the solution. Here $L$ represents the size of a cluster, $T_{L}$ the period of the lurch, $\Delta_{h}$ the time spent firing and $\Delta$ the duration of inhibition where the rebound variable $r$ is increasing. Grey regions indicate where the system is firing.

where consecutive active clusters are adjacent to each other. We suppose that to a first approximation neurons for $x \in(0, L)$ are simultaneously released from inhibition and start firing at time $t=T_{L}$. The next group with $x \in(L, 2 L)$ fires when $t=2 T_{L}$. We define the firing duration of a cluster as $\Delta_{h}$ (i.e., the time spent above $h$ ) and the duration of inhibition (time spent below $v_{r}$ before release) as $\Delta$. An illustration of this type of lurching pulse is shown in Figs. 12 and 13. An analysis of this type of solution has been given in [84]. Here it was shown that a lurching wave takes the simple (separable) form

$$
u(x, t)=W(x) Q\left(t, \min \left(t, \Delta_{h}\right)\right),
$$

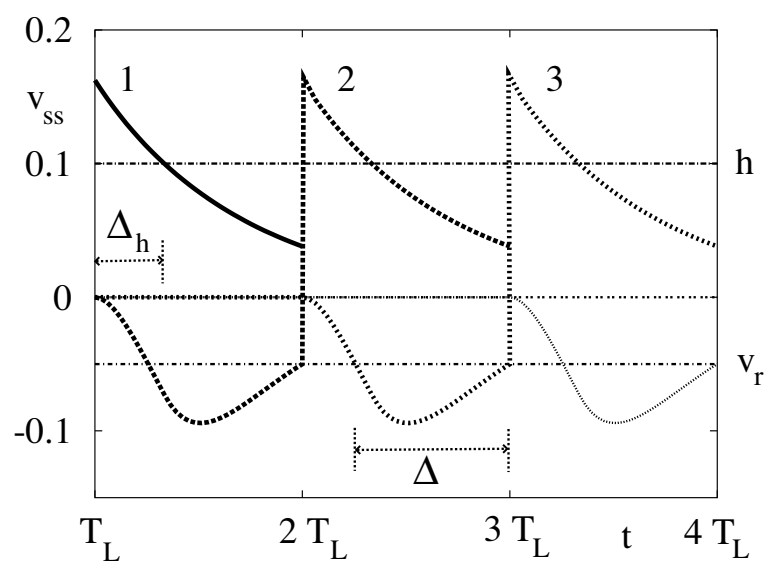

Figure 13: A plot of the analytical solution for a lurching pulse, with an $\alpha$ function synaptic response, $\eta(t)=$ $\alpha^{2} t \mathrm{e}^{-\alpha t}$. The lines labelled 1, 2, and 3 represent trajectories from neurons in adjacent clusters, with cluster 1 firing first. It is assumed that clusters can only fire once through rebound. In this example $\alpha=\sigma=g_{r}=$ $1, \tau^{+}=2, \tau^{-}=10, h=.1$, and $v_{r}=-0.05$. The numerical solution of the system of defining equations gives $\left(L, T_{L}, \Delta, \Delta_{h}\right)=(0.5,3.21,2.39,1.07)$. Note that for clarity only partial trajectories are plotted.

for $x \in(0, L)$ and $t>0$, where

$$
\begin{aligned}
Q(t, a) & =\int_{0}^{a} \eta(t-s) \mathrm{d} s, \\
W(x) & =\int_{x}^{x+L} w(y) \mathrm{d} y .
\end{aligned}
$$

The full solution is defined by periodic extension such that $u\left(x+L, t+T_{L}\right)=u(x, t)$. Hence, using (73), we have a closed form expression for $v_{\mathrm{ss}}$ in terms of the four unknowns $L, T_{L}, \Delta$ and $\Delta_{h}$. Note that if $2 L<$ $\sigma$ then $W(x)=L / 2 \sigma$ and $u(x, t)$ given by (75) is independent of $x$. Assume to a first approximation that $v_{\mathrm{ss}}(x, t)=v(0, t)$ for $x \in(0, L)$, then the four unknowns are determined by the simultaneous solution of $v_{\mathrm{ss}}\left(0, T_{L}\right)=v_{r}, v_{\mathrm{ss}}\left(0, T_{L}+\Delta_{h}\right)=h, v_{\mathrm{ss}}\left(0, T_{L}-\Delta\right)=$ $v_{r}$ and $v_{\mathrm{ss}}\left(L, T_{L}\right)=v_{r}$. Since $W(L)$ takes its maximal value for $L=\sigma / 2$ we see that there is a solution with $L=\sigma / 2$. It is convenient to introduce a lurch velocity $c=L / T_{L}$. A numerical solution of the four simultaneous equations may be used to construct $c=c\left(v_{r}\right)$, and a plot of such a solution for an alpha function synaptic response is shown in Fig. 14. Lurching waves are 


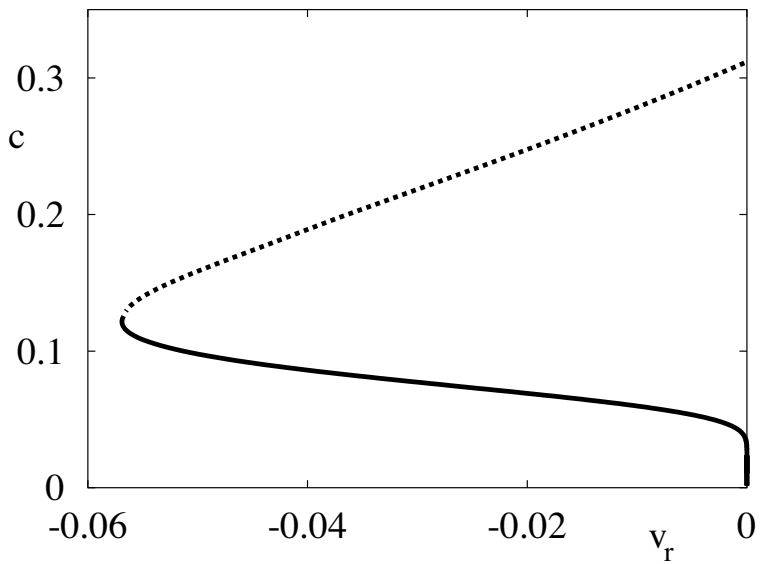

Figure 14: Speed $c=L / T_{L}$ of a solitary lurching pulse in an inhibitory network with the slow $I_{T}$ current as a function of the rebound threshold $v_{r}$. Parameters as in Fig. 13

found for $v_{r}<0$, with $c \rightarrow 0$ as $v_{r} \rightarrow 0$ (on the lower branch). Moreover, $c$ increases with decreasing $v_{r}$ and a solution is lost in a saddle-node bifurcation. Note that in the absence of the $I_{T}$ current this model network would not support any form of propagating activity. Direct numerical simulations suggest that the slow wave (lower branch) is stable. It remains an open challenge to establish this result analytically.

\section{Extension to 2D}

In specific brain regions, such as mammalian neocortex, connectivity patterns follow a laminar arrangement, with strong vertical coupling between layers. Consequently cortical activity is considered as occurring on a two dimensional plane, with the coupling between layers ensuring near instantaneous vertical propagation. Hence, it is highly desirable to obtain solutions to fully planar neural field models. We have already briefly discussed Turing instabilities in two spatial dimensions in section 3, and will not pursue this further. Rather the challenge is to ascertain whether an extra spatial dimension can lead to any fundamentally new network behaviour as compared to that in one dimension. In comparison to the studies of one dimensional networks, far less is known about be- haviour in two dimensions. Relatively recently, however, Laing and Troy [36] have developed PDE methods to study scalar neural field equations in two spatial dimensions. The focus of this work has been on the numerical construction of spatially localised (radially symmetric) solutions (via shooting methods) and their stability (via the numerical solution of an eigenvalue problem). Their work shows how circularly symmetric $N$-bumps play an important role in the formation of asymmetric $N$-bumps. By choosing radially symmetric kernels that allow for an equivalent PDE model their work also opens up the possibility for the numerical study of travelling waves $[19,85]$. However, when a local PDE approach is not possible (as is the case for synaptic footprints with compact support) one must seek solutions of the full integral neural field model directly. Thus far, the only analytical results are for radially symmetric time-independent solutions. Taylor [86] and Werner and Richter [87] have classified some of the circular and ring solutions (for Heaviside firing rate functions), and analysed the stability of solutions with respect to radial perturbations. More recent work by Folias and Bressloff [65] introduces techniques that can also handle angular perturbations.

In this section we show how some of the ideas of previous sections can be carried over to two spatial dimensions. We will limit our discussion to timeindependent localised solutions, rather than the far more challenging study of travelling waves. By focusing on the properties of a radially symmetric onebump solution we will highlight the importance of considering angular perturbations, and not just radial ones, in the determination of bump stability.

The two dimensional generalisation of (9) for a radially symmetric footprint takes the form

$$
u(\mathbf{r}, t)=\int_{\mathbb{R}^{2}} \mathrm{~d} \mathbf{r}^{\prime} w\left(\left|\mathbf{r}-\mathbf{r}^{\prime}\right|\right) \int_{0}^{\infty} \mathrm{d} s \eta(s) f \circ u\left(\mathbf{r}^{\prime}, t-s\right),
$$

where $\mathbf{r}=(r, \theta)$ and $\mathbf{r}^{\prime}=\left(r^{\prime}, \theta^{\prime}\right)$. Time-independent solutions satisfy

$$
q(\mathbf{r})=\int_{\mathbb{R}^{2}} \mathrm{~d} \mathbf{r}^{\prime} w\left(\left|\mathbf{r}-\mathbf{r}^{\prime}\right|\right) f \circ q\left(\mathbf{r}^{\prime}\right) .
$$

Linearising about the time-independent solution by 
writing $u(\mathbf{r}, t)=q(\mathbf{r})+u(\mathbf{r}) \mathrm{e}^{\lambda t}$ and working to first order in $u(\mathbf{r})$ generates the eigenvalue equation:

$$
u(\mathbf{r})=\operatorname{LT}[\eta](\lambda) \int_{\mathbb{R}^{2}} \mathrm{~d} \mathbf{r}^{\prime} w\left(\left|\mathbf{r}-\mathbf{r}^{\prime}\right|\right) f^{\prime}\left(q\left(\mathbf{r}^{\prime}\right)\right) u\left(\mathbf{r}^{\prime}\right) .
$$

As an example we consider the construction of a radially symmetric bump solution for the choice $f(u)=$ $\Theta(u-h)$. In this case we have that $q(\mathbf{r})=q(r)$, where $r=|\mathbf{r}|$, with $q(r)>h$ for $r \leq a$ and is zero otherwise. Hence, from (78)

$$
q(r)=\int_{0}^{2 \pi} \int_{0}^{a} w\left(\left|\mathbf{r}-\mathbf{r}^{\prime}\right|\right) r^{\prime} \mathrm{d} r^{\prime} \mathrm{d} \theta .
$$

This is readily evaluated using a 2D Fourier transform (equivalent to a Hankel transform) of $w(\mathbf{r})$, which we write in the form

$$
w(r)=\int_{0}^{\infty} \widetilde{w}(k) J_{0}(r k) k \mathrm{~d} k .
$$

Here $J_{\nu}(x)$ is the Bessel function of the first kind, of order $\nu$ and

$$
\widetilde{w}(k)=\int_{\mathbb{R}^{2}} \mathrm{e}^{i \mathbf{k} \cdot \mathbf{r}} w(\mathbf{r}) \mathrm{d} \mathbf{r} .
$$

Following [65] it may then be shown that substitution of (81) into (80) gives

$$
q(r)=2 \pi a \int_{0}^{\infty} \widetilde{w}(k) J_{0}(r k) J_{1}(a k) \mathrm{d} k .
$$

Using the fact that $f^{\prime}(u)=\delta(r-a) /\left|q^{\prime}(a)\right|$ means that (79) reduces to

$$
u(r, \theta)=\frac{a \operatorname{LT}[\eta](\lambda)}{\left|q^{\prime}(a)\right|} \int_{0}^{2 \pi} w\left(\left|\mathbf{r}-\mathbf{a}^{\prime}\right|\right) u\left(a, \theta^{\prime}\right) \mathrm{d} \theta^{\prime},
$$

where $\mathbf{a}^{\prime}=\left(a, \theta^{\prime}\right)$. Following [36] and [65] we look for solutions of the form $u(r, \theta)=u_{m}(r) \mathrm{e}^{i m \theta}$, where $m \in \mathbb{Z}$. In this case the radial component of the eigenfunction satisfies

$$
\begin{aligned}
\frac{u_{m}(r)}{u_{m}(a)} & =\frac{a \operatorname{LT}[\eta](\lambda)}{\left|q^{\prime}(a)\right|} \int_{0}^{2 \pi} \mathrm{d} \theta \cos (m \theta) \\
& \times w\left(\sqrt{r^{2}+a^{2}-2 r a \cos \theta}\right)
\end{aligned}
$$

where we have exploited the fact that $\int_{0}^{2 \pi} w(\mid \mathbf{r}-$ $\left.\mathbf{a}^{\prime} \mid\right) \sin (m \theta) \mathrm{d} \theta=0$. Hence, radial perturbations away from the border of the bump are completely determined by the perturbation at the bump edge (as in the one dimensional case). Setting $r=a$ in (85) generates an implicit expression for the discrete spectrum $\lambda=\lambda_{m}$, where $\lambda_{m}$ is the solution to $\mathcal{E}_{m}(\lambda) \equiv$
$\operatorname{LT}[\eta](\lambda)^{-1}-\mu_{m}=0$, with $\mu_{m} \in \mathbb{R}$ given by

$$
\mu_{m}=\frac{2 a}{\left|q^{\prime}(a)\right|} \int_{0}^{\pi} w(2 a \sin \theta) \cos (2 m \theta) \mathrm{d} \theta .
$$

We interpret $\mathcal{E}_{m}(\lambda)$ as a family of Evans functions for the two dimensional single bump. The condition for stability is thus that $\operatorname{Re}\left(\lambda_{m}\right)<0$ for all $m$. Although it is hard to find closed form expressions for $\lambda_{m}$ it is a simple matter to obtain them numerically.

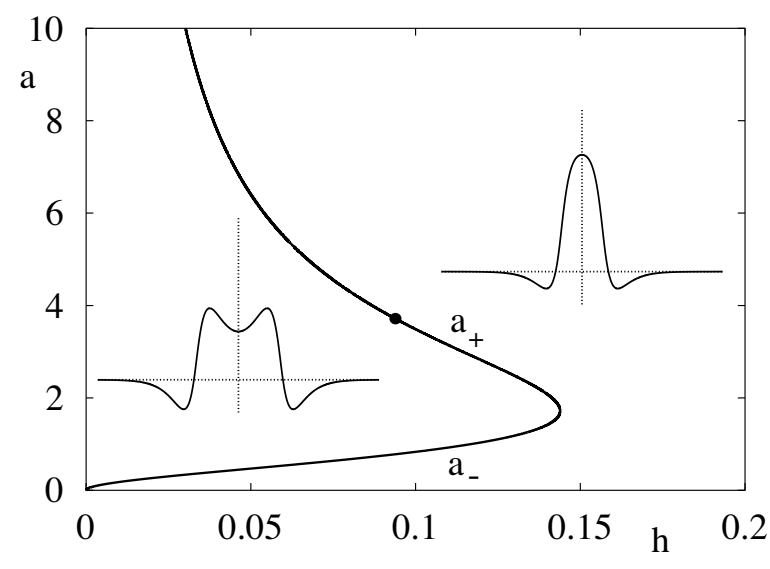

Figure 15: Bump radius $a$ as a function of threshold $h$. Note that for $h<h_{D}$ the bump solution on the branch $a_{+}$has a dimple. The point where $h=h_{D}$ on the upper branch is indicated by the filled black circle.

An evaluation of the bump solution (83) in closed form is typically only possible for special choices of $w(r)$. In fact it is easier to choose forms of $\widetilde{w}(k)$ (the 2D Fourier transform of $w(\mathbf{r}))$ that allow the use of known integral formulas involving products of Bessel functions. From the analysis of one dimensional stationary solutions we would expect to obtain bump solutions for a radially symmetric kernel of the form $w(r)=\mathrm{e}^{-r}-\mathrm{e}^{-r / 2} / 2$. Since this two dimensional Mexican hat function does not have a simple Hankel transform we make use of the approximation

$$
\frac{1}{2 \pi} \mathrm{e}^{-r} \approx \frac{2}{3 \pi}\left(K_{0}(r)-K_{0}(2 r)\right) \equiv E(r),
$$

where $K_{\nu}(x)$ is the modified Bessel function of the second kind. For computational simplicity we now work with the explicit choice $w(r)=E(r)-E(r / 2) / 4$. The factor of 4 enforces the balance condition $\int_{\mathbb{R}^{2}} w(|\mathbf{r}|) \mathrm{d} \mathbf{r}=$ 

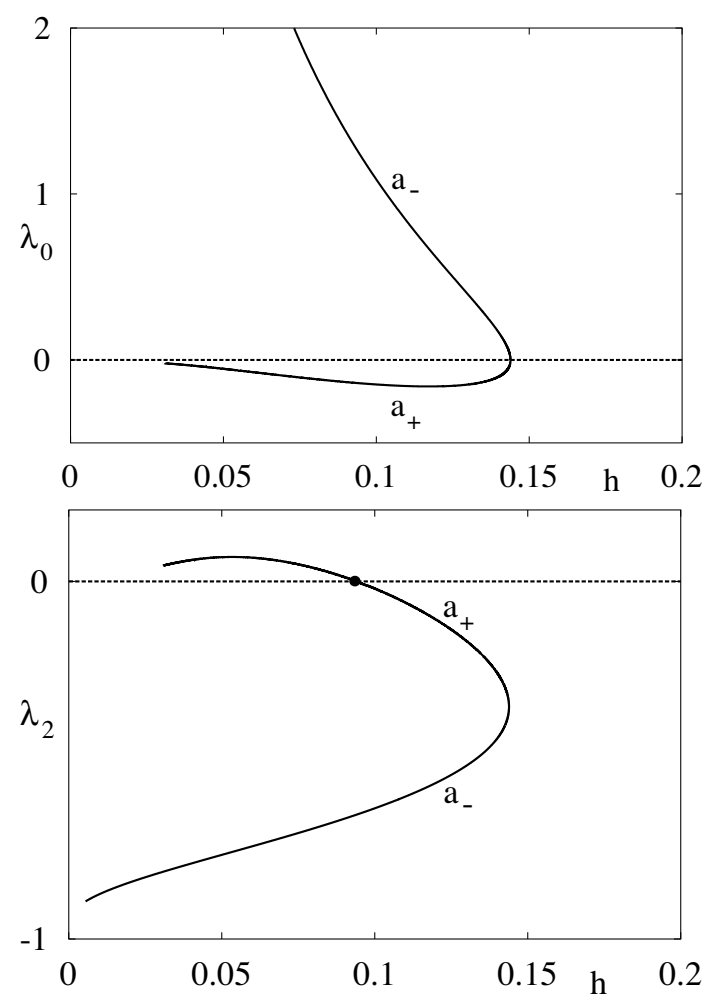

Figure 16: A plot of $\lambda_{0}$ and $\lambda_{2}$ along the solution curve of Fig. 15. We note that $\lambda_{1}=0$ for all points on the curve $a=a(h)$. Hence, although solutions on $a_{+}$ are stable to radial perturbations, for $h<h_{D}$ dimpled solutions are unstable to perturbations of the form $u_{2}(r) \cos (2 \theta)$.

0 , although this is not strictly necessary for the generation of bump solutions. Using the fact that the Hankel transform of $K_{0}(p r)$ is $H_{p}(k)=\left(k^{2}+p^{2}\right)^{-1}$ we may write

$$
\widetilde{w}(k)=\frac{2}{3 \pi}\left\{-H_{2}(k)+\frac{5}{4} H_{1}(k)-\frac{1}{4} H_{1 / 2}(k)\right\} .
$$

Substitution into (83) leads to integrals of the form

$$
\int_{0}^{\infty} \frac{J_{0}(r k) J_{1}(a k)}{k^{2}+p^{2}} \mathrm{~d} k \equiv L_{p}(a, r) .
$$

Integrals of this type are given by $[65,88]$

$$
L_{p}(a, r)=\left\{\begin{array}{ll}
\frac{1}{p} I_{1}(p a) K_{0}(p r) & r \geq a \\
\frac{1}{a p^{2}}-\frac{1}{p} I_{0}(p r) K_{1}(p a) & r<a
\end{array},\right.
$$

which allows us to compute (83) as

$$
q(r)=\frac{4 a}{3}\left(-L_{2}(a, r)+\frac{5}{4} L_{1}(a, r)-\frac{1}{4} L_{1 / 2}(a, r)\right) .
$$

The bump radius is determined by the condition $q(a)=h$. In Fig. 15 we plot the bump radius as a function of firing threshold. This clearly has the same trend as seen in the one dimensional case (cf Fig. 4). As in the one dimensional case we find two types of solution; one with $q^{\prime \prime}(0)<0$ for $h>h_{D}$ and the other with $q^{\prime \prime}(0)>0$ for $h<h_{D}$. Examples are shown in the insets of Fig. 15. On the upper branch of Fig. 15 we have plotted the point at which $q^{\prime \prime}(0)=0$, defining the transition from dimpled to non-dimpled solutions at $h=h_{D}$. In the one dimensional case no instabilities were found on the upper branch where $a=a_{+}$. However, we shall now show that in two dimensions there is the possibility of an instability on the upper branch precisely at the point where $h=h_{D}$. Consider, for example, an exponential synaptic time course $\eta(t)=\mathrm{e}^{-t}$. In this case $\operatorname{LT}[\eta](\lambda)^{-1}=1+\lambda$ and the condition for stability is simply that $\lambda_{m}<0$ for all $m$, where $\lambda_{m}=-1+\mu_{m}$. In Fig. 16 we plot $\lambda_{0}$ and $\lambda_{2}$ along the solution branch of Fig. 15 ( $\lambda_{1}$ is identically zero by rotation invariance). Hence, although the bump on the branch with $a=a_{+}$is stable to radial perturbations (since $\lambda_{0}<0$ on $a_{+}$), it is not stable to perturbations with $m=2$. Indeed $\lambda_{2}$ crosses through zero precisely at the point $h=h_{D}$ on $a_{+}$, signalling the fact that dimple solutions are unstable. From the shape of the eigenfunction $u_{2}(r) \cos 2 \theta$, plotted in Fig. 17, we would expect the bump to split in two as $h$ is decreased through $h_{D}$. This result is confirmed in [89], where a further discussion of both bump and ring instabilities can be found.

\section{Discussion}

Although it is clear that there are an increasing number of powerful mathematical techniques to choose from when studying neural field equations it is still true that such studies would benefit from contributions of a more fundamental nature. Much of the discussion in this paper has revolved around either making links between integral models and PDEs, or working with the Heaviside firing rate function. In the former case this merely side-steps the need to develop 


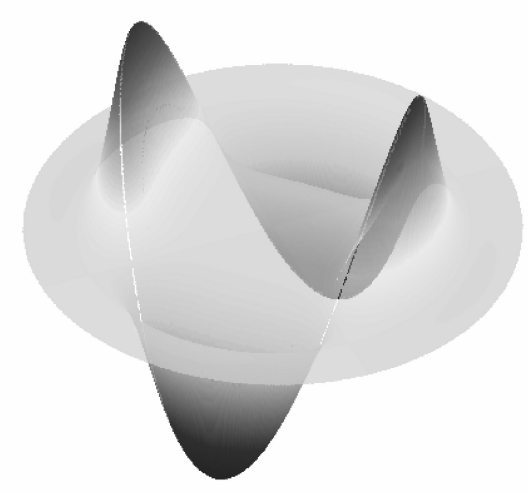

Figure 17: A plot showing the shape of the function $u_{2}(r) \cos (2 \theta)$ on the branch $a_{+}$when $h=h_{D}$. The peaks of this function occur at $r=a$. Beyond the instability point a one bump solution splits into two pieces.

general techniques for the study of nonlinear integral equations. In contrast the latter case does allow for analysis in an integral framework, but at the expense of being able to choose an arbitrary (and perhaps more realistic) firing rate function. However, it is pleasing to note that some exact results for the existence and stability of bumps have recently been obtained for non-Heaviside firing rate functions with a piecewiselinear nature $[35,90]$. For smooth firing rate functions techniques from singular perturbation theory, such as reviewed in [32], have also been useful for moving away from the Heaviside limit. Ideally however, one would like to call upon a set of new techniques that would allow the numerical continuation of solutions to nonlinear integral equations, as is commonly done for solutions to nonlinear ODEs using packages like AUTO [91]. Besides the obvious mathematical challenges of dealing with dynamic neural fields, particularly in two spatial dimensions, there are also issues to do with incorporating more biologically realistic features. We have already hinted at how to incorporate the effects of passive dendritic structures and slow ionic currents in sections 7 and 8 . However, it is also important to remember that real neural tissue is anisotropic and inhomogeneous, and that the neural field equations presented here must be modified to reflect this, as in the work of Bressloff [92]. Further- more, one must remember that the mean firing rate assumption neglects the precise details of spiking activity and as such does not take into account the effects of temporal correlations between firing events. Indeed, direct numerical simulations of spiking neural field models have uncovered a number of interesting bifurcations and dynamical phenomena, that would be ruled out in a corresponding firing rate model, e.g. $[93,61,94,80]$.

A program of work that addresses the above issues is currently underway, and will be reported on elsewhere.

\section{Acknowledgements}

I would like to thank Markus Owen for many interesting discussions held during the completion of this work. I would also like to acknowledge ongoing support from the EPSRC through the award of an Advanced Research Fellowship, Grant No. GR/R76219. 


\section{Appendix}

The coefficients $a_{0}, \ldots, a_{6}$ used in section 7 are given explicitly by

$$
\begin{aligned}
& a_{0}=\left(1+k^{2}\right)^{2}+4 \gamma k^{2} \\
& a_{1}=2\left(1+k^{2}\right) \frac{\left[\alpha(2+\gamma)+\left(1+k^{2}\right) v\right]}{\alpha v} \\
& a_{2}=\frac{2 \alpha^{2}\left(k^{2}+2 \gamma+3\right)+8 \alpha\left(1+k^{2}\right) v+\left(1+k^{2}\right)^{2} v^{2}}{\alpha^{2} v^{2}} \\
& a_{3}=2 \frac{\alpha^{2}(2+\gamma)+2 \alpha\left(3+k^{2}\right) v+2\left(1+k^{2}\right) v^{2}}{\alpha^{2} v^{3}} \\
& a_{4}=\frac{\alpha^{2}+8 \alpha v+2\left(3+k^{2}\right) v^{2}}{\alpha^{2} v^{4}} \\
& a_{5}=2 \frac{\alpha+2 v}{\alpha^{2} v^{4}} \\
& a_{6}=\frac{1}{\alpha^{2} v^{4}}
\end{aligned}
$$

\section{References}

[1] R L Beurle. Properties of a mass of cells capable of regenerating pulses. Philosophical Transactions of the Royal Society London B, 240:55-94, 1956.

[2] J S Griffith. A field theory of neural nets: I: Derivation of field equations. Bulletin of Mathematical Biophysics, 25:111-120, 1963.

[3] J S Griffith. A field theory of neural nets: II: Properties of field equations. Bulletin of Mathematical Biophysics, 27:187-195, 1965.

[4] H R Wilson and J D Cowan. Excitatory and inhibitory interactions in localized populations of model neurons. Biophysical Journal, 12:1-24, 1972.

[5] H R Wilson and J D Cowan. A mathematical theory of the functional dynamics of cortical and thalamic nervous tissue. Kybernetik, 13:55-80, 1973.

[6] J D Cowan. History of concepts and techniques. Intelligent Systems, 3:375-400, 2004.

[7] S Amari. Homogeneous nets of neuron-like elements. Biological Cybernetics, 17:211-220, 1975.

[8] S Amari. Dynamics of pattern formation in lateralinhibition type neural fields. Biological Cybernetics, 27:77-87, 1977.

[9] P 1 Nunez. Neocortical Dynamics and Human EEG Rhythms. Oxford University Press, 1995.
[10] G B Ermentrout and J D Cowan. A mathematical theory of visual hallucination patterns. Biological Cybernetics, 34:137-150, 1979.

[11] P C Bressloff, J D Cowan, M Golubitsky, P J Thomas, and $\mathrm{M}$ Wiener. Geometric visual hallucinations, Euclidean symmetry and the functional architecture of striate cortex. Philosophical Transactions of the Royal Society London B, 40:299-330, 2001.

[12] C R Laing, W C Troy, B Gutkin, and G B Ermentrout. Multiple bumps in a neuronal model of working memory. SIAM Journal on Applied Mathematics, 63:62-97, 2002.

[13] C R Laing and W C Troy. Two bump solutions of Amari-type models of working memory. Physica D, 178:190-218, 2003.

[14] M A Geise. Neural Field Theory for Motion Perception. Kluwer Academic Publishers, 1999.

[15] P Tass. Cortical pattern formation during visual hallucinations. Journal of Biological Physics, 21:177-210, 1995.

[16] K Kishimoto and S Amari. Existence and stability of local excitations in homogeneous neural fields. Journal of Mathematical Biology, 7:303-318, 1979.

[17] G B Ermentrout and J B McLeod. Existence and uniqueness of travelling waves for a neural network. Proceedings of the Royal Society of Edinburgh, 123A:461-478, 1993.

[18] D J Pinto and G B Ermentrout. Spatially structured activity in synaptically coupled neuronal networks: I. Travelling fronts and pulses. SIAM Journal on Applied Mathematics, 62:206-225, 2001.

[19] X Huang, W C Troy, Q Yang, H Ma, C R Laing, S J Schiff, and J Wu. Spiral waves in disinhibited mammalian neocortex. The Journal of Neuroscience, 24:98979902, 2004.

[20] R D Chervin, P A Pierce, and B W Connors. Periodicity and directionality in the propagation of epileptiform discharges across neortex. Journal of Neurophysiology, 60:1695-1713, 1988.

[21] D Golomb and Y Amitai. Propagating neuronal discharges in neocortical slices: Computational and experimental study. Journal of Neurophysiology, 78:1199-1211, 1997.

[22] J Y Wu, L Guan, and Y Tsau. Propagating activation during oscillations and evoked responses in neocortical slices. Journal of Neuroscience, 19:5005-5015, 1999. 
[23] R Miles, R D Traub, and R K S Wong. Spread of synchronous firing in longitudinal slices from the CA3 region of Hippocampus. Journal of Neurophysiology, 60:1481-1496, 1995.

[24] U Kim, T Bal, and D A McCormick. Spindle waves are propagating synchronized oscillations in the ferret LGNd in vitro. Journal of Neurophysiology, 74:1301-1323, 1995.

[25] M Steriade, E G Jones, and R R Línas. Thalamic Oscillations and Signalling. Wiley, New York, 1990.

[26] B W Connors and Y Amitai. Generation of epileptiform discharges by local circuits in neocortex. In P A Schwartzkroin, editor, Epilepsy: Models, Mechanisms and Concepts, pages 388-424. Cambridge University Press, 1993.

[27] G B Ermentrout and D Kleinfeld. Traveling electrical waves in cortex: Insights from phase dynamics and speculation on a computational role. Neuron, 29:33-44, 2001.

[28] C L Colby, J R Duhamel, and M E Goldberg. Oculocentric spatial representation in parietal cortex. Cerebral Cortex, 5:470-481, 1995.

[29] P S Goldman-Rakic. Cellular basis of working memory. Neuron, 14:477-485, 1995.

[30] K Zhang. Representation of spatial orientation by the intrinsic dynamics of the head-direction cell ensemble: a theory. Journal of Neuroscience, 16:2112-2126, 1996.

[31] R Ben-Yishai, L Bar-Or, and H Sompolinsky. Theory of orientation tuning in visual cortex. Proceedings of the National Academy of Sciences USA, 92:3844-3848, 1995.

[32] G B Ermentrout. Neural nets as spatio-temporal pattern forming systems. Reports on Progress in Physics, 61:353-430, 1998.

[33] S Amari. Characteristics of random nets of analog neuron-like elements. IEEE Transactions on Systems Man and Cybernetics, SMC-2:643-657, 1972.

[34] S Coombes, G J Lord, and M R Owen. Waves and bumps in neuronal networks with axo-dendritic synaptic interactions. Physica D, 178:219-241, 2003.

[35] Y Guo and C C Chow. Existence and stability of standing pulses in neural networks: I. Existence. SIAM Journal on Applied Dynamical Systems, to appear, 2005.

[36] C R Laing and W C Troy. PDE methods for nonlocal models. SIAM Journal on Applied Dynamical Systems, 2:487-516, 2003.
[37] E P Krisner. The link between integral equations and higher order ODEs. Journal of Mathematical Analysis and Applications, 291:165-179, 2004.

[38] C B Price. Traveling Turing patterns in nonlinear neural fields. Physical Review E, 55:6698-6706, 1997.

[39] P C Bressloff. Les Houches Lectures in Neurophysics, chapter Pattern formation in visual cortex, page to appear. Springer-Verlag, 2004.

[40] V K Jirsa and J A S Kelso. Spatiotemporal pattern formation in neural systems with heterogeneous connection topologies. Physical Review E, 62:8462-8465, 2000.

[41] P C Bressloff. Spatially periodic modulation of cortical patterns by long-range horizontal connections. Physica D, 185:131-157, 2003.

[42] R D Traub, J G Jefferys, and R Miles. Analysis of the propagation of disinhibition induced after-discharges along the guineau-pig hippocampal slice in vitro. Journal of Physiology, 472:267-287, 1993.

[43] K A Richardson, S J Schiff, and B J Gluckman. Control of traveling waves in the mammalian cortex. Physical Review Letters, 94:028103, 2005.

[44] O Diekmann. Thresholds and travelling waves for the geographical spread of infection. Journal of Mathematical Biology, 6:109-130, 1978.

[45] O Diekmann and H G Kaper. On the bounded solutions of a nonlinear convolution equation. Nonlinear Analysis, 2:721-737, 1978.

[46] X Chen. Existence, uniqueness, and asymptotic stability of traveling waves in nonlocal evolution equations. Advances in Differential Equations, 2:125-160, 1997.

[47] Z Chen, G B Ermentrout, and J B McLeod. Traveling fronts for a class of nonlocal convolution differential equation. Applicable Analysis, 64:235-253, 1997.

[48] F Chen. Travelling waves for a neural network. Electronic Journal of Differential Equations, 2003:1-4, 2003.

[49] M A P Idiart and L F Abbott. Propagation of excitation in neural network models. Network, 4:285-294, 1993.

[50] D Cremers and A V M Herz. Traveling waves of excitation in neural field models: equivalence of rate descriptions and integrate-and-fire dynamics. Neural Computation, 14:1651-1667, 2002.

[51] S Coombes and M R Owen. Evans functions for integral neural field equations with Heaviside firing rate function. SIAM Journal on Applied Dynamical Systems, 34:574-600, 2004. 
[52] J Evans. Nerve axon equations: IV The stable and unstable impulse. Indiana University Mathematics Journal, 24:1169-1190, 1975.

[53] L Zhang. On stability of traveling wave solutions in synaptically coupled neuronal networks. Differential and Integral Equations, 16:513-536, 2003.

[54] T Kapitula, N Kutz, and B Sandstede. The Evans function for nonlocal equations. Indiana University Mathematics Journal, 53:1095-1126, 2004.

[55] J Rubin. A nonlocal eigenvalue problem for the stability of a traveling wave in a neuronal medium. Discrete and Continuous Dynamical Systems A, 4:925-940, 2004.

[56] D J Pinto, R K Jackson, and C E Wayne. Existence and stability of traveling pulses in a continuous neuronal network. SIAM Journal on Applied Dynamical Systems, page to appear, 2005.

[57] E Folias and P C Bressloff. Stimuluslocked waves and breathers in an excitatory neural network. preprint, 2005. http:/ / www.math.utah.edu/ bresslof/publications/052abs.html.

[58] A Hutt. Effects of nonlocal feedback on traveling fronts in neural fields subject to transmission delay. Physical Review E, 70:052902, 2004.

[59] P C Bressloff and S E Folias. Front-bifurcations in an excitatory neural network. SIAM Journal on Applied Mathematics, 65:131-151, 2004.

[60] D J Pinto and G B Ermentrout. Spatially structured activity in synaptically coupled neuronal networks: II. Lateral inhibition and standing pulses. SIAM Journal on Applied Mathematics, 62:226-243, 2001.

[61] B S Gutkin, C R Laing, C C Chow, G B Ermentrout, and C L Colby. Turning on and off with excitation: the role of spike-timing asynchrony and synchrony in sustained neural activity. Journal of Computational Neuroscience, 11:121-134, 2001.

[62] J Rubin and W Troy. Sustained spatial patterns of activity in neuronal populations without recurrent excitation. SIAM Journal on Applied Mathematics, 64:16091635, 2004.

[63] M Camperi and X J Wang. A model of visuospatial working memory in prefrontal cortex: recurrent network and cellular bistability. Journal of Computational Neuroscience, 5:383-405, 1998.
[64] C P Fall, T Lewis, and J Rinzel. Background activity dependent properties of a network model for working memory that incorporates cellular bistability. Biological Cybernetics, this issue, 2005.

[65] S E Folias and P C Bressloff. Breathing pulses in an excitatory neural network. SIAM Journal on Applied Dynamical Systems, 3:378-407, 2004.

[66] S Coombes and M R Owen. Bumps, breathers, and waves in a neural network with spike frequency adaptation. Physical Review Letters, to appear, 2005.

[67] V K Jirsa and H Haken. Field theory of electromagnetic brain activity. Physical Review Letters, 77:960-963, 1996.

[68] P C Bressloff. New mechanism for neural pattern formation. Physical Review Letters, 76:4644-4647, 1996.

[69] P C Bressloff and S Coombes. Physics of the extended neuron. International Journal of Modern Physics B, 11:2343-2392, 1997.

[70] V K Jirsa and H Haken. A derivation of a macroscopic field theory of the brain from the quasi-microscopic neural dynamics. Physica D, 99:503-526, 1997.

[71] J A S Kelso, S L Bressler, S Buchanan, G C Deguzman, M Ding, A Fuchs, and T Holroyd. A phase-transition in human brain and behaviour. Physics Letters A, 169:134144, 1992.

[72] P A Robinson, C J Rennie, J J Wright, H Bahramali, E Gordon, and D 1 Rowe. Prediction of electroencephalographic spectra from neurophysiology. Physical Review E, 63:021903, 2001.

[73] J J Wright and R R Kydd. The electroencephalogram and cortical neural networks. Network, 3:341-362, 1992.

[74] D T J Liley, P J Cadusch, and M P Dafilis. A spatially continuous mean field theory of electrocortical activity. Network, 13:67-113, 2002.

[75] M L Steyn-Ross, D A Steyn-Ross, J W Sleigh, and D R Whiting. Theoretical predictions for spatial covariance of the electroencephalographic signal during the anesthetic-induced phase transition: Increased correlation length and emergence of spatial self-organization. Physical Review E, 68:021902, 2003.

[76] M Enculescu. A note on traveling fronts and pulses in a firing rate model of a neuronal network. Physica $D$, 196:362-386, 2004.

[77] A Hutt, M Bestehorn, and T Wennekers. Pattern formation in intracortical neuronal fields. Network, 14:351368, 2003. 
[78] R Curtu and B Ermentrout. Pattern formation in a network of excitatory and inhibitory cells with adaptation. SIAM Journal on Applied Dynamical Systems, 3:191-231, 2004.

[79] P C Bressloff and S Coombes. Dynamics of stronglycoupled spiking neurons. Neural Computation, 12:91$129,2000$.

[80] C Laing and C C Chow. Stationary bumps in networks of spiking neurons. Neural Computation, 13:1473-1494, 2001.

[81] G D Smith, C L Cox, S M Sherman, and J Rinzel. Fourier analysis of sinusoidally driven Thalamocortical relay neurons and a minimal integrate-and-fire-orburst model. Journal of Neurophysiology, 83:588-610, 2000.

[82] J Rinzel, D Terman, X J Wang, and B Ermentrout. Propagating activity patterns in large-scale inhibitory neuronal networks. Science, 279:1351-1355, 1998.

[83] D H Terman, G B Ermentrout, and A C Yew. Propagating activity patterns in thalamic neuronal networks. SIAM Journal on Applied Mathematics, 61:1578-1604, 2001.

[84] S Coombes. Dynamics of synaptically coupled integrate-and-fire-or-burst neurons. Physical Review E, 67:041910, 2003.

[85] C R Laing. Spiral waves in nonlocal equations. SIAM Journal on Applied Dynamical Systems, to appear, 2005.

[86] J G Taylor. Neural 'bubble' dynamics in two dimensions: foundations. Biological Cybernetics, 80:393-409, 1999.

[87] H Werner and T Richter. Circular stationary solutions in two-dimensional neural fields. Biological Cybernetics, 85:211-217, 2001.

[88] G N Watson. A treatise on the theory of Bessel functions. Cambridge University Press, 1952.

[89] S Coombes and M R Owen. Bumps, rings, and spots in a two-dimensional neural field. in preparation, 2005.

[90] Y Guo and C C Chow. Existence and stability of standing pulses in neural networks: II. Stability. SIAM Journal on Applied Dynamical Systems, to appear, 2005.

[91] E J Doedel, A R Champneys, T R Fairgrieve, Y A Kuznetsov, B Sandstede, and X J Wang. AUTO97 continuation and bifurcation software for ordinary differential equations. 1997. Available from http://indy.cs.concordia.ca/auto/main.html.
[92] P C Bressloff. Traveling fronts and wave propagation failure in an inhomogeneous neural network. Physica D, 155, 2001.

[93] D Golomb and G B Ermentrout. Effects of delay on the type and velocity of travelling pulses in neuronal networks with spatially decaying connectivity. Network, 11:221-246, 2000.

[94] P H Chu, J D Milton, and J D Cowan. Connectivity and the dynamics of integrate-and-fire networks. International Journal of Bifurcation and Chaos, 4:237-243, 1994. 